\title{
IMPLEMENTATION OF WIND TURBINE CONTROLLERS
}

\author{
D.J.Leith \\ W.E.Leithead \\ Department of Electronic \& Electrical Engineering, \\ University of Strathclyde, \\ GLASGOW G1 1QE, U.K.
}

\begin{abstract}
Three of the important, generic, implementation issues encountered when developing controllers for pitch-regulated constant-speed wind turbines are considered, namely, (1) accommodation of the strongly nonlinear rotor aerodynamics; (2) automatic controller start-up/shut-down; and (3) accommodation of velocity and acceleration constraints within the actuator. Both direct linearisation and feedback linearisation methods for accommodating the nonlinear aerodynamics are investigated and compared. A widely employed technique for accommodating the nonlinear aerodynamics, originally developed on the basis of physical insight, is rigorously derived and extended to cater for all wind turbine configurations. A rigorous stability analysis of controller start-up is presented for the first time and novel design guidelines are proposed which can significantly reduce the power transients at controller start-up. The relation to anti-wind-up is noted and several aspects of an existing wind-turbine controller start-up strategy are observed to be novel in the anti-wind-up context. Restrictive position, velocity and acceleration constraints may all be present in wind turbines and the dynamic behaviour of the actuator cannot be neglected. A novel, and quite general, anti-wind-up method, based on the startup strategy, is proposed which caters for all these circumstances. The separate strategies for resolving the implementation issues are combined to achieve an elegant controller realisation which accommodates all the implementation issues in an integrated manner. The importance of adopting an appropriate controller realisation is considerable and is illustrated for a $300 \mathrm{~kW}$ wind turbine. The implementation issues encountered in this paper are, of course, not confined to wind turbines but are of wider concern.
\end{abstract}

\section{Introduction}

Wind turbine technology has undergone rapid development in recent years in response to widespread demands for the increased use of renewable sources of energy. The standard commercial design of wind turbine is a horizontal-axis grid-connected up-wind machine with a rating of approximately $300 \mathrm{~kW}$ to $500 \mathrm{~kW}$ (medium-scale). It is anticipated that the next generation of wind turbines, which are presently being developed, will include large-scale designs with a rating of around 1 MW. The rotor usually has two or three blades, and in pitch regulated machines the pitch angle of either the full span of the blades, or just the outer tips, can be varied. The control design task for constant-speed pitch-regulated machines is to exploit this capability in order to regulate the power output whilst minimising the load transients and thereby reducing fatigue damage. The objectives and design of the SISO control system are discussed fully by Leithead et al. (1991a, b, 1992a).

In this paper, three of the important implementation issues encountered, when developing controllers for pitch-regulated constant-speed wind turbines, are investigated, namely,

1. Compensation of the strongly nonlinear rotor aerodynamics.

2. Automatic controller start-up/shut-down.

3. Accommodation of velocity and acceleration constraints within the actuator.

These issues are, of course, not confined to wind turbines but are of wider concern.

The first implementation issue relates to the aerodynamic behaviour of the turbine blades. Whilst a linearised plant representation is typically employed during the synthesis of the controller transfer function, a wind turbine is a nonlinear system; in particular, the aerodynamic behaviour is highly nonlinear. Since the role of the turbine rotor in converting wind energy into mechanical energy is a central one, the nonlinear behaviour of the rotor exerts a substantial influence on the characteristics of the whole wind turbine and cannot be neglected (see, for example, Leithead et al. 1991a, 1992a).

The second implementation issue relates to the wind turbine operational requirements. Below a certain 'rated wind speed', the power generated is less than the turbine rating and no control action is required. However, when the wind speed rises above rated, the power output is regulated at the rated value of the turbine, 'rated power', by suitably adjusting the pitch angle of the rotor blades. Hence, it is 
necessary to automatically start-up and shut-down the controller as the wind speed fluctuates. (In very low wind speeds, there is insufficient energy to sustain power generation and the turbine is shut down. In very high wind speeds, the turbine is shut down to prevent damage. Start-up and shut-down of the entire turbine, rather than just the controller, is not considered here). The controller has integral action to ensure rejection of steady wind disturbances and suitable low frequency shaping (Leithead et al. 1991a, 1992a) to ensure rejection of gusts (ramp-like increases or decreases in wind speed which persist over several seconds). Consequently, controller start-up requires to be treated with some care to avoid prolonged transients and minimise the loads on the wind turbine.

The third implementation issue relates to the actuator physical constraints, neglected during the controller transfer function synthesis. In low wind speeds, when the sensitivity of the aerodynamic torque to changes in the pitch angle is lowest, it is often necessary to move the blades of the turbine rapidly in order to achieve adequate power regulation. The hard limits on the accelerations (or torques) and velocities, internal to the actuator, are, therefore, often encountered during normal operation near rated wind speed. At present, the impact of the hard actuator limits on stability and performance, in the context of wind turbine applications, has received little consideration.

Although strategies adopted to resolve these implementation issues are often proprietary in nature, strategies, which adequately address the implementation issues, are reported in the open literature by Leithead et al. (1989, 1990a, b, 1991a, 1992a). (Their effectiveness has been confirmed in field trials (Leithead \& Agius 1991)). However, these strategies were developed on the basis of physical insight, supported by simulation studies, and a more rigorous analytic approach remains desirable. A reexamination and analytic investigation of the implementation issues is further warranted by the recent development of high performance nonlinear controllers (Leith \& Leithead 1995a, b, c, 1996a, b), which operate the control system at its limits to a much greater extent than conventional linear controllers, and by the progression of the wind turbine rating from medium-scale to large-scale with the consequent trend towards a considerable tightening of the physical constraints on the control system.

The paper is organised as follows. Section two contains a brief discussion of those aspects of wind turbine modelling and control relevant to the present context and section three a review of strategies for the resolution of the implementation issues considered in this paper. The rigorous compensation of the nonlinear aerodynamics is considered in section four, controller start-up/shut-down in section five and accommodation of actuator velocity and acceleration constraints in section six. The individual strategies to resolve the implementation issues, considered separately in sections four, five and six, are combined in section seven to produce an integrated controller realisation and the performance improvement achieved is illustrated by an example. Finally, in section eight the conclusions are summarised.

\section{Wind Turbine Control Preliminaries}

In this paper, the controller implementation issues are mainly illustrated with respect to a mediumscale $300 \mathrm{~kW}$ two-bladed grid-connected up-wind constant-speed full-span pitch-regulated wind turbine which is dynamically representative of commercial machines of its class. A block diagram representation of the system dynamics is depicted in figure 1.

Although there is no such thing as the 'wind speed' experienced by a wind turbine, since the rotor is subject to a spatially and temporally distributed wind field, it may be considered to experience an effective wind speed which, in some sense, is an average over the rotor disc. (It should be noted that this makes a direct measurement of wind speed impossible). The aerodynamic torque, T, depends nonlinearly on both the pitch angle, $\mathrm{p}$, and the effective wind speed, V, as in figure $2 \mathrm{a}$; that is,

$$
\mathrm{T}=\mathrm{T}(\mathrm{p}, \mathrm{V})
$$

(Throughout this paper it is assumed that $\mathrm{T}$ is differentiable as required). For each effective wind speed, $\mathrm{V}$, above rated wind speed, the rated aerodynamic torque, $\mathrm{T}_{\mathrm{o}}$, is attained at a unique pitch angle, $\mathrm{p}_{\mathrm{V}}$. These pitch angles together with their corresponding effective wind speeds define the locus of equilibrium operating points, $\left(\mathrm{p}_{\mathrm{V}}, \mathrm{V}\right)$, of the system. Locally to a specific equilibrium operating point, $\left(\mathrm{p}_{\mathrm{V}}, \mathrm{V}\right)$, the nonlinearity (1) may be linearised as depicted in figure $2 \mathrm{~b}$, where $\delta$ indicates perturbations about the nominal values. The partial derivatives, at the equilibrium points, of the aerodynamic torque with respect to pitch angle and wind speed, $\partial \mathrm{T} / \partial \mathrm{p}$ and $\partial \mathrm{T} / \partial \mathrm{V}$ respectively, are tabulated in table 1 . It can be seen that, over the operational envelope of the wind turbine, the behaviour of the aerodynamic torque varies considerably. 
The combined dynamics of the drive-train, generator and power transducer, G, are essentially linear and, together, are modelled by the transfer function

46460.9

$$
\mathrm{G}(\mathrm{s})=\frac{}{\left(\mathrm{s}^{5}+81.27 \mathrm{~s}^{4}+3683.90 \mathrm{~s}^{3}+120773.6 \mathrm{~s}^{2}+1474504.0 \mathrm{~s}+36857450.0\right)}
$$

The actuator position is physically constrained to the range $[-2.0,60.0]$ degrees and the velocity to the range $[-10.0,10.0]$ degrees/second. Since the pitch angle control demand is constrained to the range $[0.0,45.0]$ degrees, the former constraint can be neglected and the actuator dynamics, A, represented by the block diagram depicted in figure 3. The dynamic terms, $M_{0}, M_{1}$ and $T$, are modelled by the transfer functions

$$
\mathrm{M}_{\mathrm{o}}(\mathrm{s})=1.00 ; \quad \mathrm{M}_{1}(\mathrm{~s})=14.0 ; \quad \mathrm{T}(\mathrm{s})=50.0 /(\mathrm{s}+50.0)
$$

Within the velocity constraint, the actuator is effectively linear with bandwidth $20.7 \mathrm{r} / \mathrm{s}$. Hence, for control purposes, its dynamics, $\mathrm{A}_{\mathrm{u}}$, when unsaturated, can be modelled by $\hat{\mathrm{A}}_{\mathrm{u}}$ (s) where

$$
\hat{\mathrm{A}}_{\mathrm{u}}(\mathrm{s})=\frac{20.7}{\mathrm{~s}+20.7}
$$

Although a similar representation of the system dynamics was adopted in Leithead \& Agius (1991) and Leith \& Leithead (1995a, b, c, 1996a, b), a different representation had been adopted previously (Leithead et al. 1989, 1990a, 1991a, 1992a) whereby an additional term representing dynamic inflow (also known as induction lag) was included between the aerodynamics and the drive-train dynamics in figure 1. The dynamic inflow is modelled as a simple lead-lag but its representation is subject to considerable uncertainty and the frequencies of the pole and zero depend on the wind speed. However, since the pole and zero are at very low frequencies, the dynamic inflow has little impact on the synthesis of the controller transfer function, except, perhaps, requiring an adjustment in the gain, and the controller synthesis models need not include it. (Note, it does not necessarily follow that it has little impact on the controller implementation and can be omitted altogether from the control design models). In addition, no evidence for the presence of dynamic inflow could be discerned during experimental control studies (Leithead \& Agius 1991), raising some doubt concerning its influence on the system dynamics. Hence, as in Leithead \& Agius (1991) and Leith \& Leithead (1995a, b, c, 1996a, b), the dynamic inflow may be omitted in the context of controller transfer function synthesis provided the gain is confirmed from measured data.

The aerodynamic behaviour of the wind turbine is highly nonlinear and strongly dependent on wind speed. However, its representation, described above, is very basic and subject to considerable uncertainty. In addition, the aerodynamics may change over time due to contamination of the blade surfaces. Consequently, a good gain margin, in conjunction with a good phase margin, is particularly important in order to achieve adequate stability margins. If adequate stability margins are not achieved, the system must sometimes destabilise, although not necessarily become unstable, in which case the wind turbine would experience large load fluctuations. Because of the complexity of the interaction of the rotor with the wind-field, it is not possible to quantify the uncertainty in the aerodynamic models but practical experience indicates that $10 \mathrm{~dB}$ is an appropriate gain margin and roughly 60 degrees the appropriate phase margin.

A controller transfer function, $\mathrm{C}(\mathrm{s})$, is synthesised, for the equilibrium operating point corresponding to a wind speed of $12 \mathrm{~m} / \mathrm{s}$, such that

$$
\mathrm{C}(\mathrm{s})=\mathrm{C}_{\text {outer }}(\mathrm{s}) \mathrm{C}_{\text {inner }}(\mathrm{s})
$$

with

$$
(\mathrm{s}+1.5)(\mathrm{s}+1.6)
$$

$$
\mathrm{C}_{\text {inner }}(\mathrm{s})=7.50 \frac{\mathrm{s}(\mathrm{s}+0.3)(\mathrm{s}+3.7)}{\mathrm{s}+1.5)(\mathrm{s}+1.6)}
$$




$$
\mathrm{C}_{\text {outer }}(\mathrm{s})=133.60 \frac{\left(\mathrm{s}^{2}+7.243 \mathrm{~s}+38.637\right)\left(\mathrm{s}^{2}+1.5 \mathrm{~s}+104.04\right)\left(\mathrm{s}^{2}+6 \mathrm{~s}+416.16\right)}{(\mathrm{s}+20)(\mathrm{s}+50)\left(\mathrm{s}^{2}+11 \mathrm{~s}+104.04\right)\left(\mathrm{s}^{2}+10 \mathrm{~s}+416.16\right)\left(\mathrm{s}^{2}+65.8 \mathrm{~s}+2209\right)}
$$

The Bode plot of the open-loop transfer function is shown in figure 4. It exhibits the low frequency shaping to improve disturbance rejection referred to in section 1, high frequency roll-off to reduce actuator activity and notches at $2 \mathrm{P}$ and $4 \mathrm{P}$ (where $\mathrm{P}$ is the rotational speed of the rotor) to reduce actuator activity and reduce enhancement of the loads induced by spectral peaks, due to rotational sampling of the wind-field, at these frequencies. The cross-over frequency is $1.87 \mathrm{r} / \mathrm{s}$ and the gain and phase margins are $10.2 \mathrm{~dB}$ and 51 degrees, respectively.

Both the quantitative and qualitative behaviour exhibited by medium and large scale machines can differ substantially. In particular, due to the increased physical size and inertia of the blades, the bandwidth of the actuator of a large-scale machine may be reduced to such an extent that it is similar to the closed-loop system bandwidth. In addition, the actuator constraints may become tighter and it may be required to operate close to the limits over a wide range of wind speeds. Accordingly, in this paper the controller implementation issues are, in addition to the $300 \mathrm{~kW}$ machine, also occasionally illustrated with reference to a 1 MW wind turbine (Rogers \& Leithead 1994) with an actuator bandwidth of $2.5 \mathrm{r} / \mathrm{s}$ and a speed limit of 7 degrees/second.

\section{Review of Previous Strategies}

The success of a strategy adopted to resolve the implementation issues may be assessed against two criteria: first, the reduction or elimination of large transient loads, as measured by the generated power; second, the extent to which the stability margins are maintained without compromising performance (adequate stability margins must, of course, be attained following the adoption of a particular strategy; if necessary by revising the linear control algorithm). Strategies devised previously to resolve the implementation issues are briefly reviewed below.

\subsection{Compensating for the Nonlinear Aerodynamics}

Fluctuations in the wind speed induce variations in the aerodynamic torque and it is emphasised that the wind speed, and hence the torque, vary rapidly and continuously, in a stochastic manner, over the whole operational envelope. The requirement is to devise a means of accommodating the nonlinear aerodynamic behaviour such that a fixed linear control algorithm may be employed over the whole operational envelope. The discussion is confined, for the moment, to the issue of accommodating the nonlinear aerodynamics.

One solution to this task, proposed by Leithead et al. (1990a, b, 1991a), is illustrated in figure 5a. The controller is augmented with a nonlinear gain corresponding to the reciprocal of the sensitivity of

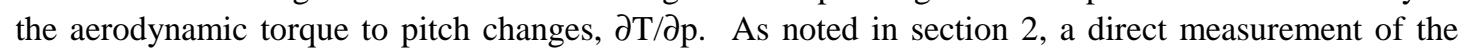
wind speed cannot be employed to adjust this gain. However, irrespective of actuator bandwidth, the controller should attempt to ensure that the pitch angle is appropriately set at each wind speed. Consequently, assuming that the pitch angle is close to $\mathrm{p}_{\mathrm{v}}$, the need for a measurement of wind speed is avoided; that is, the wind speed may be inferred directly from the pitch angle. (Of course, a degree of error, adding to general system uncertainty, can be expected). It is known that the performance of this approach to aerodynamic compensation is strongly dependent on the positioning of the nonlinear gain, see figure 6, and that the appropriate position is after the main controller dynamics but before the pure integrator term (Leithead et al 1990a, b, 1991a). (Since the controller has integral action, the differentiation is trivially absorbed by the controller).

The approach depicted in figure 5a, or approaches which are essentially equivalent, are widely employed and are remarkably effective on current medium-scale machines. However, whilst engineering insight provides a basis for understanding the effectiveness of this approach, a rigorous analysis is lacking and it is uncertain whether the method is appropriate for other wind turbine configurations; in particular, for large-scale machines which, owing to physical restrictions, can have very low bandwidth actuators.

\subsection{Controller Start-Up/Shut-Down}


A common approach to controller start-up is to simply freeze the controller integral action when below rated operation is detected; that is, when the demanded pitch angle of the turbine blades falls below a specified threshold value (zero degrees here), the accumulation sum in the integral calculation is suspended. However, this approach is not always effective in preventing large start-up transients; for example, the power output during a typical start-up, depicted in figure 7, rises to over $600 \mathrm{~kW}$ (twice the rated value) when this method is employed on the $300 \mathrm{~kW}$ machine. Intuitively, whilst this approach prevents the pure integrator in the controller from adopting an inappropriate state during below-rated operation, it does not cater for the low frequency pole in the controller with which the prolonged start-up transients are associated.

Leithead et al. (1990a, b, 1991a, 1992a) propose an alternative start-up technique whereby a minor feedback loop is introduced within the controller which mimics the action of the physical wind turbine and switches in to permit the controller to continue operating below rated wind speed (equivalent to a negative pitch angle demand). Designing the controller, such that $\mathrm{C}_{\text {outer }}$ may be interpreted as an approximation to the inverse of the plant dynamics and $\mathrm{C}_{\text {inner }}$ may be interpreted as an approximation to the open-loop system, the minor loop may be implemented in a straightforward manner by partitioning the controller as depicted in figure 8 . In the minor feedback loop, the high bandwidth filter, F, attenuates any switching transients and the feedback gain approximates the plant aerodynamic gain. Since the minor feedback loop may become active whilst the actual pitch angle is greater than zero, the feedback gain is scheduled, with respect to the actual pitch angle, in a similar manner to the controller nonlinear gain. The start-up transients are substantially reduced with this strategy, as indicated in figure 7. When the dynamic inflow is thought to contribute significantly to the aerodynamics, it is natural to assume that a component, corresponding to the dynamic inflow, should be included in $\mathrm{C}_{\text {outer }}$ (Leithead et al. 1990a, b). However, superior performance is obtained when it is omitted (Leithead et al. 1991a, 1992a). Hence, since it makes little impact on the control design task, either with respect to synthesis of the controller transfer function or design of the strategy to cater for the implementation issues, the dynamic inflow can be omitted altogether from the control design models as in (Leithead \& Agius 1991, Leith \& Leithead 1995a, b, c, 1996a, b) and here. The distinction between the dynamics of $\mathrm{C}_{\text {outer }}$ and $\mathrm{C}_{\mathrm{inner}}$ is now that the controller is partitioned such that the slow dynamics are contained in $\mathrm{C}_{\mathrm{inner}}$.

However, whilst the operation of the foregoing strategy for controller start-up might be understood intuitively, a rigorous analysis, particularly of stability, is lacking and the relation between these methods, developed for a specific application, and existing control design techniques, developed in a more general context, has not been investigated.

\subsection{Accommodating Actuator Velocity/Acceleration Saturation}

In the context of wind turbine applications, the presence of hard actuator limits is known (Leithead et al. 1991a) to degrade performance by causing a reduction in stability. However, this reduction is much less (Leithead et al. 1991a) when the nonlinear controller gain, which caters for the nonlinear aerodynamic behaviour, is scheduled on the demanded pitch angle, as in Leithead et al. (1992a), rather than the actual pitch angle. In the context of medium-scale wind turbines, when the bandwidth of the actuator is relatively large, the pitch angle demand is an equally effective choice of scheduling variable in comparison to actual pitch angle. (When the actuator bandwidth is smaller, as might be the case in a large-scale wind turbine, choosing the pitch angle demand may be less appropriate). Of course, the scheduling variable for the minor feedback loop gain must then also be scheduled on pitch angle demand (Leithead et al. 1992a). The nonlinear controller gain is constant below rated wind speed, being held at the value for zero degrees, and, since the minor feedback loop only becomes active in below rated wind speed, the feedback loop gain can, and should, also be constant.

Other than the above, the impact of the hard actuator limits on stability and performance has received little consideration. Traditionally, the control specification requires that the controller avoids operating the actuator at its hard limits; for example, the permitted standard deviation of the constrained quantities may be suitably restricted. Nevertheless, in order to achieve satisfactory performance, it may be necessary to relax such restrictions, particularly for large-scale machines (Rogers \& Leithead 1994). However, an analysis of the impact of hard actuator limits, particularly on the stability margins of wind turbines, is lacking.

The complete strategy, proposed by Leithead et al. (1991a, 1992a) to resolve the implementation issues, is depicted in figure 9.

\section{Compensating for the Nonlinear Aerodynamics}




\subsection{Gain-Scheduling}

The gain-scheduling approach to compensation of the nonlinear aerodynamics is to incorporate the reciprocal of the aerodynamic 'gain', $\partial \mathrm{T} / \partial \mathrm{p}\left(\mathrm{p}_{\mathrm{v}}, \mathrm{V}\right)$, within the controller and schedule this gain with respect to a variable which parameterises the locus of equilibrium operating points. (Since scheduling on a direct measurement of wind speed is impossible, the pitch angle may be employed provided the pitch angle is always sufficiently close to $p_{\mathrm{V}}$ ). When the system is sufficiently weakly nonlinear (or, equivalently, the operating point of the system remains sufficiently close to the locus of equilibrium operating points and varies sufficiently slowly), the stability of the gain-scheduled nonlinear system may be inferred from the stability of the members of the family of linear systems consisting of the linearisations of the nonlinear system at each equilibrium operating point (see, for example, Desoer \& Vidyasagar 1975, Shamma \& Athans 1990, Khalil \& Kokotovic 1991).

However, the wind speed fluctuations are highly stochastic and the operating point of the wind turbine varies rapidly and continuously over the whole operating envelope. Whilst the bandwidth of the linearised closed-loop system is typically about $3 \mathrm{r} / \mathrm{s}$, the operating point might cover its full range, corresponding to an order of magnitude or greater change in the aerodynamic gain, in a few seconds. Moreover, large, rapid fluctuations in wind speed are common, in particular gusts; that is, steady increases or decreases in the wind speed which persist for relatively long periods and produce substantial and prolonged perturbations from equilibrium. Hence, a priori, the system cannot be assumed to be weakly nonlinear. A simple test, for a system with integral control action, is to compare the behaviour of the closed-loop system with the nonlinear controller gain positioned before and after the integrator (Leith \& Leithead 1996a); for weakly nonlinear systems, the behaviours should not differ substantially. From figure 6, it is clear that an assumption of weak nonlinearity is not warranted. Consequently, the emphasis must be on the nonlinear and non-local behaviour and performance of the controller.

Leith \& Leithead (1996a) propose that, with regard to a gain-scheduled controller, appropriate nonlocal behaviour may be ensured by adopting a controller realisation which satisfies an extended local linear equivalence condition. (Briefly, the gain-scheduled controller is obtained by interpolating between the members of a family of linear controllers designed for specific operating points. The extended local linear equivalence condition requires that the linearisation of the gain-scheduled controller should match the appropriate member of the linear family at every operating point, both equilibrium operating points and non-equilibrium operating points). For the wind turbine controller, this is achieved by placing the scheduled gain after the main controller dynamics but before the pure integrator term and by incorporating a model of the actuator dynamics and its inverse ${ }^{1}$ in the controller as depicted in figure $5 b$ (Leith \& Leithead 1996a). When the actuator bandwidth is sufficiently large, figure $5 \mathrm{~b}$ reduces to figure 5a; that is, to the approach of Leithead et al. (1990a, b, 1991a).

However, for appropriate non-local behaviour, it is required that the complete system, not just the controller, must satisfy the extended local linear equivalence condition. Since this is not the case here, analytic support for the nonlinear gain-scheduled controller is, really, only provided by the above discussion for sufficiently slow perturbations which are local to the locus of equilibrium operating points and not for prolonged non-local perturbations. A nonlinear analysis of the nonlinear gainscheduled controller is required.

\subsection{Separability of the Nonlinear Aerodynamics}

It is known that the dependence, on pitch angle and wind speed, of the aerodynamic pitching moment of the turbine blades can be explicitly separated (Leithead et al. 1992b). The same methodology may be applied to the aerodynamic torque (Leithead et al. 1995). Since the aerodynamic torque is constant along the locus of equilibrium operating points, the partial derivatives of $\mathrm{T}$ are related on the locus by

$$
\frac{\partial \mathrm{T}}{\partial \mathrm{V}}\left(\mathrm{p}_{\mathrm{V}}, \mathrm{V}\right)=-\frac{\mathrm{dp}(\mathrm{V})}{\mathrm{dV}} \frac{\partial \mathrm{T}}{\partial \mathrm{p}}\left(\mathrm{p}_{\mathrm{V}}, \mathrm{V}\right)
$$

\footnotetext{
${ }^{1}$ When a first order model of the actuator is sufficient, the inverse model is combined with the pure integrator within the controller to attain a proper realisation. When a higher order model is necessary an approximate, realisable, inverse model is employed (Leith \& Leithead 1996a).
} 
where $\mathrm{dp}(\mathrm{V}) / \mathrm{dV}$ is the rate of change, with respect to wind speed, of pitch angle along the locus. It follows that $(\mathrm{h}(\mathrm{p})-\mathrm{g}(\mathrm{V}))$ is constant on the locus of equilibrium operating points provided $\mathrm{h}$ and $\mathrm{g}$ satisfy the conditions

$$
\begin{aligned}
& \text { dh } \begin{array}{lll}
\partial \mathrm{T} & \mathrm{dg} & \partial \mathrm{T}
\end{array} \\
& \frac{-}{\mathrm{dp}}=\frac{-}{\partial \mathrm{p}}\left(\mathrm{p}_{\mathrm{V}}, \mathrm{V}\right) ; \quad \frac{-}{\mathrm{dV}}=-\frac{-}{\partial \mathrm{V}}\left(\mathrm{p}_{\mathrm{V}}, \mathrm{V}\right)
\end{aligned}
$$

for all $\left(\mathrm{p}_{\mathrm{v}}, \mathrm{V}\right)$. Of course, $(10)$ defines, within a constant, $\mathrm{h}(\bullet)$ for all pitch angles and $\mathrm{g}(\bullet)$ for all above rated wind speeds. Without loss of generality, $(\mathrm{h}(\mathrm{p})-\mathrm{g}(\mathrm{V}))$ can be chosen to be zero on the locus. Hence, locally to the locus of equilibrium operating points,

$$
\mathrm{T}(\mathrm{p}, \mathrm{V}) \equiv \tau(\varepsilon) ; \quad \varepsilon=\mathrm{h}(\mathrm{p})-\mathrm{g}(\mathrm{V})
$$

for some function $\tau$ such that,

$$
\tau(0)=\mathrm{T}_{0} ; \quad \mathrm{d} \tau / \mathrm{d} \varepsilon(0)=1
$$

since, locally to any point $\left(\mathrm{p}_{\mathrm{V}}, \mathrm{V}\right)$ on the locus,

$$
\tau\left(\mathrm{h}\left(\mathrm{p}_{\mathrm{V}}+\delta \mathrm{p}\right)-\mathrm{g}(\mathrm{V}+\delta \mathrm{V})\right) \approx \underset{\mathrm{T}}{\mathrm{T}}+\frac{\partial \mathrm{T}}{\partial \mathrm{p}}\left(\mathrm{p}_{\mathrm{V}}, \mathrm{V}\right) \delta \mathrm{p}+\frac{\partial \mathrm{T}}{\partial \mathrm{V}}\left(\mathrm{p}_{\mathrm{V}}, \mathrm{V}\right) \delta \mathrm{V}
$$

$$
\approx \mathrm{T}\left(\mathrm{p}_{\mathrm{v}}+\delta \mathrm{p}, \mathrm{V}+\delta \mathrm{V}\right)
$$

The relationship of the aerodynamic torque to pitch demand can, therefore, be represented by (11) locally to the locus of equilibrium operating points with $g(\bullet)$ embodying the time-varying nonlinear dependence on wind speed and $\tau(\bullet)$ representing the nonlinear dependence on displacement from the locus of equilibrium operating points (see figure $2 \mathrm{c}$ ).

The preceding analysis indicates that the local separability, embodied by (11), is a quite general property which pertains to a broad class of nonlinear regulation problems. It is stressed that (11) is valid non-locally in the sense that it is not confined to describing the behaviour about a single equilibrium operating point but rather describes the behaviour in a neighbourhood about the complete locus of equilibrium operating points. In the present application, the neighbourhood of the locus of equilibrium points for which (11) is an adequate representation of the nonlinear aerodynamics is substantial (Leithead et al. 1995). For example, for the $300 \mathrm{~kW}$ wind turbine it can be seen from figure 10 that (11) is accurate when the aerodynamic torque, and so power, is kept below 2.5 times its rated value and that $\tau(\bullet)$ is almost linear with unity gain in this region. There is no difficulty in achieving this requirement with existing control systems (see, for example, figure 6).

The separability of the nonlinear aerodynamics is not unexpected because there are underlying physical reasons why the representation (11) should hold for a wide neighbourhood for all wind turbines. The aerodynamic torque largely stems from the outer third of the rotor but, in this region, the velocity of the blade is much greater than the wind velocity. It follows that the direction of the wind velocity, relative to the blades, changes almost linearly as the wind speed varies but its magnitude changes little. Hence, the aerodynamic torque is largely a function of the angle of attack of the wind on the outer third of the blades, which is simply the difference in the direction of the relative velocity of the wind and the pitch angle.

\subsection{Direct Linearisation}

Owing to the separability of the nonlinear aerodynamics, the compensation problem may be reformulated as one of linearising the SISO memoryless nonlinearity, $h(p)$, whilst accommodating the dynamics of the actuator (which lie between the controller and the nonlinearity). It follows immediately that this is achieved by the approach depicted in figure $11 \mathrm{a}$, where $\hat{\mathrm{h}}^{-1}, \hat{\mathrm{A}}_{\mathrm{u}}, \hat{\mathrm{A}}_{\mathrm{u}}^{-1}$ are suitable approximations to, respectively, $\mathrm{h}^{-1}$, the actuator dynamics and the inverse actuator dynamics. (Exact linearisation is achieved when there is no approximation error). Of course, $\mathrm{h}^{-1}(\bullet)$, must exist (which requires $\mathrm{dh} / \mathrm{dp}(\bullet)$, and so $\partial \mathrm{T} / \partial \mathrm{p}\left(\mathrm{p}_{\mathrm{v}}, \mathrm{V}\right)$, to be monotonic).

The strategy of figure 11a is, however, not unique. For example, from figure 11a,

$$
\hat{\mathrm{p}}=\hat{\mathrm{h}}^{-1}(\hat{\phi})
$$

and, differentiating with respect to time,

$$
\dot{\hat{\mathrm{p}}}=\frac{1}{\frac{\mathrm{d} \hat{\mathrm{h}}}{\mathrm{d} \hat{\mathrm{p}}}(\hat{\mathrm{p}})} \dot{\hat{\phi}}
$$


Hence, it follows that figure 11a may be reformulated as depicted in figure 11b. (The differentiation operator in this formulation can be incorporated without difficulty into the linear controller owing to the integral action of the latter). The non-local linearisation, figure $11 \mathrm{~b}$, is essentially identical in form to the local linearisation, figure $5 \mathrm{~b}$, derived in section 4.1 using gain-scheduling methods. Hence, the gain-scheduling approach to accommodating the nonlinear aerodynamics, whilst derived on the basis of local analysis, in fact achieves, albeit inadvertently, non-local linearisation. Moreover, when the actuator bandwidth is sufficiently large, figure $11 \mathrm{~b}$ reduces to figure 5a, thereby, providing a rigorous theoretical basis for the approach of Leithead et al (1990a, b, 1991a, 1992a). However, when the actuator bandwidth is low, the approach of Leithead et al. (1990a, b, 1991a, 1992a) may not be appropriate. The performance of the representative $1 \mathrm{MW}$ machine, with and without compensation for the slow actuator dynamics in the aerodynamic linearisation, is illustrated by figure 12; a rather substantial difference, with a maximum of about $400 \mathrm{~kW}$, is evident in the generated power.

In practice, when implementing the linearisations of figure 11 , nominal models for $A_{u}$ and $A_{u}^{-1}$ are employed. Furthermore, the representation of the aerodynamic behaviour of the turbine rotor is subject to considerable uncertainty with $\mathrm{h}(\bullet)$ only inexactly known. With regard to the sensitivity to errors in $\hat{\mathrm{A}}_{\mathrm{u}}, \hat{\mathrm{A}}_{\mathrm{u}}^{-1}, \hat{\mathrm{h}}^{-1}$ and $\mathrm{d} \hat{\mathrm{h}} / \mathrm{d} \hat{\mathrm{p}}$, the linearisations, in figure $11 \mathrm{a}$ and figure $11 \mathrm{~b}$, are equivalent and it suffices to consider the latter alone. In figure $11 \mathrm{~b}$, the actuator dynamics and aerodynamics are directly inverted so that, nominally, $\phi$ equals $\hat{\phi}$. To achieve effective linearisation, this inversion must retain reasonable accuracy for all admissible signals, $\hat{\phi}$, despite the approximation errors. It is necessary, therefore, to assess the impact of these on the effectiveness of the linearisation; that is, to assess the robustness.

In the present application, and in general, a requirement on the controller is to strongly attenuate high frequency signals; for example, to protect the actuator from high frequency wind disturbances and measurement noise and to avoid exciting unmodelled plant dynamics. Moreover, all physical actuators attenuate high frequency signals as must $\hat{\mathrm{A}}_{\mathrm{u}}$. Hence, the spectrum of $\hat{\phi}$ may be assumed to have little energy at high frequencies. Furthermore, in order to meet the demanding performance specification, the actuator behaviour must be reasonably well known over the range of admissible control signals; that is, for a linear actuator, its transfer function must be known over the control bandwidth of the system. (Straightforward laboratory or field tests can be employed to determine the actuator characteristics). It follows that $\hat{\mathrm{A}}_{\mathrm{u}}{ }^{-1}$ may be selected such that $\mathrm{A}_{\mathrm{u}} \hat{\mathrm{A}}_{\mathrm{u}}{ }^{-1}$, essentially, has unity gain for the admissible signals, $\hat{p}$; that is, for a near linear actuator, discrepancies between the transfer functions of $\hat{\mathrm{A}}_{\mathrm{u}}^{-1}$ and $\mathrm{A}_{\mathrm{u}}^{-1}$ are confined to high frequencies where the spectrum of $\hat{\mathrm{p}}$ has little energy. Therefore,

$$
\mathrm{p}=\hat{\mathrm{p}}+\varepsilon
$$

where $\varepsilon$ is small; indeed, it is inherent to the controller specification that the controller is designed to ensure $\varepsilon$ is small.

Since

$$
\frac{1}{\frac{d \hat{h}}{d \hat{p}}(\hat{p})}=\frac{K(\hat{p})}{\frac{d h}{d \hat{p}}(\hat{p})} ; \quad K(\hat{p})=\frac{\frac{d h}{d \hat{p}}(\hat{p})}{\frac{d \hat{h}}{d \hat{p}}(\hat{p})}
$$

figure $11 \mathrm{~b}$ may be reformulated, using (14) and (15), as figure 11c. Hence, figure 11c, and so the complete system, may be interpreted to be entirely linear except for a gain-scheduled controller which satisfies the extended linear equivalence condition (Leith \& Leithead 1996a). For such a system, the dynamic properties may be inferred from those of the family of linear systems obtained by linearising about the equilibrium operating points. Hence, provided the nonlinearity of $K(\hat{p})$ is not excessively strong, variation in $\mathrm{K}(\hat{\mathrm{p}})$ from unity is easily accommodated by the stability margins of the members of the family of linear systems. Consequently, the strategy for accommodating the nonlinear aerodynamics depicted in figure $11 \mathrm{~b}$ or, equivalently, figure $11 \mathrm{a}$, is robust for the class of applications considered.

\subsection{Feedback Linearisation}

Two specific strategies to accommodate the nonlinear aerodynamics are derived directly in the preceding section. However, this objective may also be achieved, within a more general framework, by 
considering the actuator dynamics in combination with the nonlinear aerodynamics to be a nonlinear dynamic system and applying feedback linearisation (see, for example, Isidori 1989, Slotine \& Li 1991).

Let $\mathrm{h}: \Re^{\mathrm{n}} \rightarrow \mathfrak{R}$ be a smooth (sufficiently differentiable) scalar function and $\mathbf{f}, \mathbf{g}: \mathfrak{R}^{\mathrm{n}} \rightarrow \mathfrak{\Re}^{\mathrm{n}}$ be smooth vector fields on $\Re^{\mathrm{n}}$. The Lie derivative, or directional derivative, with respect to $\mathbf{f}$ is the scalar function defined by $L_{f} h=\nabla h \cdot f$. Higher order Lie derivatives are defined recursively by $L_{f}^{i} h=L_{f}{ }^{o} L_{f}^{i-1} h, L_{f}{ }^{o} h=h$; similarly, $\mathrm{L}_{\mathrm{g}} \mathrm{L}_{\mathrm{f}} \mathrm{h}=\nabla \mathrm{L}_{\mathrm{f}} \mathrm{h} \cdot \mathrm{g}$. Consider the SISO nonlinear dynamic system

$$
\mathbf{x}=\mathbf{f}(\mathbf{x})+\mathbf{g}(\mathbf{x}) \mathrm{z} ; \quad \mathrm{p}=\mathbf{C x} ; \quad \phi=\mathrm{h}(\mathrm{p})=\mathrm{H}(\mathbf{x})
$$

where, in the present application, $\mathrm{p}$ is the pitch angle, $\mathrm{z}$ is the input applied to the actuator and $\mathbf{f}, \mathbf{g}$ define the (perhaps nonlinear) actuator dynamics. When $\mathrm{z}$ satisfies

$$
\mathrm{z}=\frac{-\mathrm{L}_{\mathbf{f}}^{\mathrm{r}} \mathrm{H}+\mathrm{v}}{\mathrm{L}_{\mathbf{g}} \mathrm{L}_{\mathbf{f}}^{\mathrm{r}-1} \mathrm{H}}
$$

where $r$ is the relative degree of the system (defined by $\mathrm{L}_{\mathrm{g}} \mathrm{L}_{\mathrm{f}}^{\mathrm{i}}=0$ for $0 \leq \mathrm{i}<\mathrm{r}-1, \mathrm{~L}_{\mathrm{g}} \mathrm{L}_{\mathrm{f}}^{\mathrm{r}-1} \neq 0$ ), the nonlinear system, (18), is linearised in the sense that

$$
\phi^{(\mathrm{r})}=\mathrm{v}
$$

where $\phi^{(\mathrm{r})}$ denotes $\mathrm{d}^{(\mathrm{r})} \phi / \mathrm{dt}^{(\mathrm{r})}$.

In order to confine the linearisation to the nonlinear aerodynamics, thereby avoiding inversion of the actuator dynamics, $\mathrm{v}$ is chosen to be

$$
\mathrm{V}=\mathbf{C} \dot{\mathbf{x}}^{(\mathrm{r})}
$$

where $\hat{\mathbf{x}}=\mathbf{f}(\hat{\mathbf{x}})+\mathbf{g}(\hat{\mathbf{x}}) \mathrm{u}$ with boundary condition $\mathbf{C} \hat{\mathbf{x}}(0)=\phi(0)$, and $\mathrm{u}$ is the input to the linearised system.

Although, it is, of course, straightforward to extend the analysis to accommodate higher order actuator dynamics, assume the actuator dynamics may be adequately represented by the first order system

$$
\dot{\mathrm{p}}=-\mathrm{a}^{-1} \mathrm{p}+\mathrm{a}^{-1} \mathrm{z}
$$

with $\mathrm{a}^{-1} \neq 0$; that is, in (18), $\mathbf{x}=\mathrm{p}, \mathrm{f}(\mathbf{x})=-\mathrm{a}^{-1} \mathrm{p}, \mathrm{g}(\mathbf{x})=\mathrm{a}^{-1}, \mathrm{C}=1$. In practice, a first order representation of the actuator is often adequate since a high gain feedback loop is typically employed internally to ensure that the actuator output follows its input. The first order Lie derivatives of (22) are

$$
\mathrm{L}_{\mathrm{g}} \mathrm{H}=\frac{\mathrm{dh}}{\mathrm{dp}} \mathrm{a}^{-1}, \quad \mathrm{~L}_{\mathrm{f}} \mathrm{H}=-\frac{\mathrm{dh}}{\mathrm{dp}} \mathrm{a}^{-1} \mathrm{p}
$$

From (23a), the relative degree, $r$, of the system is unity when $\frac{d h}{d p} \neq 0, a^{-1} \neq 0$. The former condition is necessary for the wind turbine to be controllable whilst, from (22), the latter is satisfied. Hence, applying (20) and (21) to (22),

$$
\phi=\mathrm{v}=\dot{\hat{\mathbf{x}}}=-\mathrm{a}^{-1} \phi+\mathrm{a}^{-1} \mathrm{u}
$$

Hence, (19) becomes

$$
\mathrm{z}=\left(\frac{\mathrm{dh}}{\mathrm{dp}} \mathrm{p}-\mathrm{h}(\mathrm{p})+\mathrm{u}\right) / \frac{\mathrm{dh}}{\mathrm{dp}}
$$

which corresponds to the block diagram representation depicted in figure 13a. Clearly, in figure 13a, a positive feedback loop, which essentially cancels with the actuator internal feedback to leave it openloop, encloses the actuator.

In contrast to the direct linearisation approach of section 4.3 , the feedback linearisation approach requires that measurements of certain quantities be available. When the actuator dynamics are first order, a measurement of the pitch angle is required. More generally, when the actuator transfer function is $\mathrm{n}^{\text {th }}$ order, measurements of the first $(\mathrm{n}-1)$ derivatives of the pitch angle are required for exact linearisation. For example, when the actuator has the second-order transfer function $1 /\left(a_{2} s^{2}+a_{1} s+1\right)$, the linearising feedback becomes

$$
\mathrm{z}=\left(-\mathrm{a}_{2} \frac{\mathrm{d}^{2} \mathrm{~h}}{\mathrm{dp}^{2}} \dot{\mathrm{p}}^{2}+\frac{\mathrm{dh}}{\mathrm{dp}} \mathrm{p}-\mathrm{h}(\mathrm{p})+\mathrm{u}\right) / \frac{\mathrm{dh}}{\mathrm{dp}}
$$

An alternative linearisation is obtained by incorporating integral action into the actuator such that

$$
\mathrm{a} \ddot{\mathrm{p}}+\dot{\mathrm{p}}=\mathrm{z}
$$

For these augmented dynamics 


$$
\mathrm{L}_{\mathrm{g}} \mathrm{H}=0 ; \quad \mathrm{L}_{\mathrm{g}} \mathrm{L}_{\mathbf{f}} \mathrm{H}=\frac{\mathrm{dh}}{\mathrm{dp}} \mathrm{a}^{-1} ; \quad \mathrm{L}_{\mathbf{f}}^{2} \mathrm{H}=-\frac{\mathrm{dh}}{\mathrm{dp}} \mathrm{a}^{-1} \dot{\mathrm{p}}+\frac{\mathrm{d}^{2} \mathrm{~h}}{\mathrm{dp}^{2}}(\dot{\mathrm{p}})^{2} ; \quad \mathrm{v}=-\frac{\mathrm{dh}}{\mathrm{dp}} \mathrm{a}^{-1} \dot{\mathrm{p}}+\mathrm{a}^{-1} \dot{\mathrm{u}}
$$

Hence, with the same conditions as in the previous linearisation, the relative degree is 2 and

$$
\dot{\mathrm{z}}=\frac{-\mathrm{a} \frac{\mathrm{d}^{2} \mathrm{~h}}{\mathrm{dp}^{2}}(\dot{\mathrm{p}})^{2}+\dot{\mathrm{u}}}{\frac{\mathrm{dh}}{\mathrm{dp}}}
$$

which corresponds to the block diagram representation depicted in figure 13b. In general, when the actuator transfer function is $n^{\text {th }}$ order, measurements of the first $n$ derivatives of the pitch angle are required with this formulation. When the actuator bandwidth is sufficiently high, the term, $a \frac{d^{2} h}{d p^{2}}(\dot{p})^{2}$, in (27) arising from the actuator dynamics may be neglected, and figure $13 \mathrm{~b}$ reduces to figure $5 \mathrm{a}$, the approach of Leithead et al. (1990a, b, 1991a, 1992a). However, the term, $a \frac{d^{2} h}{{d p^{2}}^{2}}(\dot{p})^{2}$, may be significant when the actuator bandwidth is low. Indeed, it provides an exact statement of the circumstances under which the bandwidth of the actuator may be considered to be low. (It is noted that this measure depends, as might be expected, not only on the bandwidth of the actuator but also on the strength of the nonlinearity and the rate at which the operating point changes). Since $\frac{\mathrm{d}^{2} \mathrm{~h}}{\mathrm{dp}^{2}}$ is negative for wind turbines, the term in (27) arising from the actuator dynamics is always positive. It follows that its omission introduces a bias in the controller velocity demand which, in turn, introduces a bias in the power output that is most pronounced when the pitch velocity is large (see, for example, figure 12).

\subsection{Relationship Between Direct \& Feedback Linearisation}

Strategies for accommodating the nonlinear aerodynamics are derived both by direct linearisation (section 4.3) and by application of feedback linearisation (section 4.4). Clearly, it is necessary to determine their relationship, if any, to each other.

Consider, firstly, the approach of figure 11a, for which, for first order actuator dynamics,

$$
\mathrm{a} \hat{\phi}+\hat{\phi}=\mathrm{u} ; \hat{\mathrm{p}}=\hat{\mathrm{h}}^{-1}(\hat{\phi}) ; \mathrm{z}=\hat{\mathrm{p}}+\mathrm{a} \hat{\mathrm{p}}
$$

It follows immediately that

$$
\begin{aligned}
& \mathrm{a} \dot{\hat{\phi}}=\mathrm{a} \frac{\mathrm{d} \hat{\mathrm{h}}}{\mathrm{d} \hat{\mathrm{p}}} \dot{\hat{\mathrm{p}}}=(\mathrm{u}-\hat{\phi}) \\
& \mathrm{z}=\left(\frac{\mathrm{d} \hat{\mathrm{h}}}{\mathrm{d} \hat{\mathrm{p}}} \hat{\mathrm{p}}-\hat{\mathrm{h}}(\hat{\mathrm{p}})+\mathrm{u}\right) / \frac{\mathrm{d} \hat{\mathrm{h}}}{\mathrm{d} \hat{\mathrm{p}}}
\end{aligned}
$$

Neglecting measurement noise and uncertainties in the actuator dynamics and the aerodynamics, it can be seen that (30) is identical to (24) and, thus, figures 11a and 13a are nominally equivalent. Secondly, it may be shown for the approach of figure $11 \mathrm{~b}$

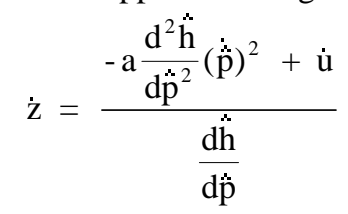

Consequently, figures $11 \mathrm{~b}$ and $13 \mathrm{~b}$ are, also, nominally equivalent. Therefore, all four schemes, depicted in figures 11 and 13 , are nominally equivalent.

Nevertheless, the formulations are not equivalent with regard to several important implementation issues.

1. The approach of figure $13 \mathrm{~b}$ requires measurements of both the pitch angle and the pitch rate. Rate measurements are, in general, more difficult to obtain than position measurements and are often rather noisy (which impacts on both performance and stability). Consequently, this approach is less attractive than, for example, that of figure 13a for which a measurement of the pitch angle alone suffices. Of course, the extension of the approach of figure 13a to accommodate higher order 
actuator dynamics (when, for example, a first-order representation of the actuator dynamics is inadequate over the control bandwidth) also requires derivative measurements (see section 4.4). Under these circumstances, the approaches based on feedback linearisation may be unsuitable and the direct linearisations of figure 11 might be preferred.

2. In wind turbine applications, $\mathrm{dh} / \mathrm{dp}$ is readily obtained from $\partial \mathrm{T} / \partial \mathrm{p}$ along the locus of equilibrium points. The function $\mathrm{h}^{-1}$, required for the formulation in figure $11 \mathrm{a}$ is then calculated as

$$
\mathrm{h}^{-1}(\mathrm{p})=\int_{0}^{\mathrm{p}} \frac{1}{\frac{\mathrm{dh}}{\mathrm{ds}}(\mathrm{s})} \mathrm{ds}
$$

with suitable boundary conditions. However, the approach of figure $11 \mathrm{~b}$ avoids this step and is, therefore, more direct for wind turbine applications than that of figure 11a.

3. When the actuator bandwidth is much greater than that of the controlled system, such that the feedback term, $a \frac{d^{2} h}{{d p^{2}}^{2}}(\dot{p})^{2}$, is small, the approaches of figures $11 \mathrm{~b}$ and $13 \mathrm{~b}$ reduce to the existing approach of Leithead et al. (1990a, b, 1991a, 1992a). For low bandwidth actuators, however, the approach of figure $11 \mathrm{~b}$ requires the availability of an adequate actuator model. Whilst this information is often available, or easily determined, the approach of figure 13a avoids altogether any need for detailed actuator knowledge provided that a measurement of pitch angle is available and the actuator exhibits first order behaviour over the control bandwidth. Furthermore, the latter approach can accommodate actuators whose characteristics vary slowly over time (see, for example, Desoer \& Vidyasagar 1975, Shamma \& Athans 1990, Khalil \& Kokotovic 1991 for theoretical results relating to the stability of slowly-varying linear and nonlinear systems).

4. The approach of figure 13a encloses the actuator in a positive feedback which, essentially, cancels with the actuator internal feedback leaving it open-loop; under nominal conditions, the negative feedback of $\hat{\phi}$ ensures that $\phi$ is dynamically linearly related to u. However, the linearity of the negative feedback is dependent on mutual cancellation, via the open-loop actuator dynamics, of the nonlinear functions, $\hat{\mathrm{h}}$ and $\frac{\mathrm{d} \hat{\mathrm{h}}}{\mathrm{d} \hat{\mathrm{p}}}$. Significant nonlinearities, which can be expected to influence the foregoing mutual cancellation in a complex manner, are, typically, present internally within real actuators. It follows that this approach can be expected to be rather less robust, in general, with respect to the actuator nonlinearities, than the other linearisation approaches (which benefit from the linearising action on the actuator of its internal high gain feedback).

Whilst the linearisation approaches which have been derived are nominally equivalent, they differ with respect to a variety of implementation issues. The foregoing discussion clearly indicates that the choice of the appropriate implementation of the linearisation is, in general, strongly application dependant. With regard to wind turbine applications, the conventional feedback linearisation approach of figure 13a is judged to be unsuitable owing to the requirement for a positive feedback loop around the actuator, with consequent robustness implications. The alternative formulation, figure 13b, is also judged to be unsuitable owing to the requirement for a measurement of pitch rate.

\section{Controller Start-Up/Shut-Down 5.1 Stability Analysis of Controller Start-up/Shut-Down}

In order to facilitate stability analysis, the controller start-up strategy of Leithead et al. (1990a, b, 1991a, 1992a), figure 8, is reformulated as depicted in figure 14. The two formulations are equivalent (ignoring, for the moment, the controller terms which compensate for the aerodynamic nonlinearity). The Small Gain theorem, and criteria derived from it such as the Circle Criterion, (see, for example, Desoer \& Vidyasagar 1975) may be employed to provide straightforward sufficiency conditions for the stability of the nonlinear closed-loop system with the controller start-up/shut-down strategy of figure 14 . In order to apply small gain analysis, the controlled system in figure 14 is reformulated as a SISO linear time-invariant block with transfer function $\mathrm{C}_{\text {inner }}\left(\mathrm{F}_{-} \mathrm{C}_{\text {outer }} \mathrm{GA}_{\mathrm{u}}\right) /\left(1+\mathrm{C}_{\text {inner }} \mathrm{F}\right)$ and nonlinear feedback provided by a saturation element with a lower limit of zero. The nonlinear saturation element is an 
operator with induced $L_{2}$ norm in the range $(0,1]$; therefore, by direct application of the Small Gain theorem, the closed-loop system is stable provided the magnitude of the transfer function $\mathrm{C}_{\text {inner }}\left(\mathrm{F}-\mathrm{C}_{\text {outer }}\right.$ $\left.\mathrm{GA}_{\mathrm{u}}\right) /\left(1+\mathrm{C}_{\mathrm{inner}} \mathrm{F}\right)$ does not exceed unity. From figure 15 , it is clearly seen that this condition is satisfied for the representative $300 \mathrm{~kW}$ machine when the controller start-up strategy of figure 14 is employed.

An assessment of stability robustness is also necessary and may be performed rigorously by replacing $G$ by $G(1+\Gamma)$, reformulating the system in figure 14 as a MIMO linear block with one feedback via $\Gamma$ and a second via the saturation element, and then applying small gain analysis once again. However, the rigour of this approach is counter-balanced by its inherent conservativeness which greatly restricts the utility of the stability margins thereby derived. Consequently, it is important that less conservative results are obtained. This may be achieved using harmonic balance techniques (see, for example, Atherton 1982) at the cost of reduced rigour. The dominant source of uncertainty typically lies in the aerodynamic behaviour of the turbine blades and may be considered largely as a gain uncertainty. Since the describing function of a real memoryless nonlinear gain is real, the gain margin is indicated to be a useful measure of robustness which may be assessed by increasing the plant gain and analysing the stability of the closed-loop system. With the controller start-up approach of figure 14 the $300 \mathrm{~kW}$ machine is determined to possess a gain margin of $10 \mathrm{~dB}$; that is, the gain margin of the linear open-loop system is preserved.

\subsection{Design Guidelines}

The controller start-up/shut-down approach of Leithead et al. (1990a, b, 1991a, 1992a) introduces additional design freedoms into the controller; that is, a variety of parameters which require to be selected appropriately. Leithead et al. (1991a, 1992a) suggest, on the basis of physical insight, that the closed-loop transfer function of the start-up/shut-down loop within the controller should be similar to that of the overall closed-loop system. Whilst this guideline gives rise to acceptable performance in many cases, it can be seen from figure 7 that, although the approach of Leithead et al. (1991a, 1992a) greatly reduces the power transients at start-up, these transients remain significantly larger than those experienced during continuous above-rated operation at low wind speeds; for example, around 40\% (40 $\mathrm{kW}$ ) higher in figure 7 . Consequently, design guidelines to achieve improved performance are desirable.

The dynamics of the wind turbine drive-train introduce a lag between changes in the wind speed and the corresponding changes in the power output; that is, when the wind speed rises above rated, there is a lag before the power output responds and control action is resumed. It follows that the start-up performance might be improved by anticipating the transition from below to above rated power generation and, thereby, compensating somewhat for the dynamics of the drive-train. Predictive action may be achieved by augmenting the $\mathrm{C}_{\text {outer }}$ block with a filter, $\mathrm{P}$, possessing suitable phase lead. In order to recover the controller transfer function for above-rated operation, the inverse of this filter must be included in the $\mathrm{C}_{\text {inner }}$ block (the modification to the characteristics of the start-up minor feedback loop is negligible). It is required that both the filter transfer function, $\mathrm{P}(\mathrm{s})$, and its inverse, $\mathrm{P}^{-1}(\mathrm{~s})$, are stable and both $\mathrm{C}_{\text {outer }} \mathrm{P}$ and $\mathrm{C}_{\text {inner }} \mathrm{P}^{-1}$ are realisable. For the $300 \mathrm{~kW}$ machine, a simple filter with transfer function $(s+0.75) /(s+1.5)$ introduces appropriate phase lead and reduces the start-up power transient by over 50 $\mathrm{kW}$ in figure 7 in comparison with the conventional approach of Leithead et al. (1991a, 1992a). The start-up transient is now well within the range of power fluctuation experienced during normal aboverated operation at low wind speeds; that is, extending present design guidelines to include appropriate shaping of the partitioned controller transfer functions can lead to a substantial improvement in start-up performance.

\subsection{Relationship to Anti-wind-up}

In the context of linear analysis, generation of rated power requires the pitch angle to be negative when the wind speed is below the rated. However, with regard to the nonlinear plant, the actual pitch angle is physically constrained to be positive and a negative pitch demand by the controller corresponds to below rated operation. The control action is suspended on encountering the lower limit on the pitch angle, which the blades can adopt, and reactivated by movement of the blade pitch angle off this limit. This interpretation enables a connection to be made between the, apparently rather specific, wind turbine controller start-up/shut-down problem and the general field of constrained control.

With regard to constrained control design, approaches which directly account for the presence of actuator constraints have been devised using optimal control theory (Frankena \& Sivan 1979, Garcia et al. 1989), Lyapunov methods (Gutman \& Hagander 1985), invariant set theory (Wredenhagen \& Belanger 1994) and others (for example, Sussman \& Sontag 1994). However, in practice the most 
widespread approach involves first designing a linear controller, ignoring control input constraints, and then adding some form of compensation which minimises any adverse effects of the constraints on closed-loop performance (Kothare et al. 1994). Whilst a great variety of (sometimes rather ad hoc) compensation methods have been devised (see, for example, Fertik \& Ross 1967, Shinsky 1967,1988, Buckley 1971, Astrom \& Wittenmark 1984, Hanus et al. 1987, Campo \& Morari 1990), many of the techniques can be accommodated within more general approaches (Walgama \& Sternby 1990, Kothare et al. 1994). Historically, performance degradation due to actuator saturation has often been considered in the context of PI/PID controllers and has been attributed to so-called integrator (or reset) 'wind-up' (early references include Shinsky 1967, Fertik \& Ross 1967). In qualitative terms (see, for example, Astrom \& Wittenmark 1984), when the actuator is saturated the control feedback loop is broken and the plant no longer responds to the controller. However, the pure integrator in the controller continues to be driven by the control error and its output increases in magnitude or 'winds-up'. Consequently, on returning to unsaturated operation transients develop which can lead to a loss of performance and instability. Wind-up, as described above, is only exhibited by a non-stable element such as an integrator (Astrom \& Wittenmark 1984). However, performance is also known to be degraded by stable dynamic elements with sufficiently slow dynamics (Doyle et al. 1987, Campo \& Morari 1990). Nevertheless, compensation methods for reducing the performance deterioration associated with hard actuator limits are widely referred to as 'anti-wind-up' techniques and this term is, therefore, also adopted here.

The transients associated with wind turbine controller start-up may be attributed to some form of 'wind-up' within the controller, whilst operating below rated, and start-up strategies for reducing these transients may be considered within the anti-wind-up context. General results from the field of constrained control may then be applied to wind turbine controller start-up and, similarly, solutions developed for the latter problem may be directly generalised to a broad class of constrained control problems. The formulation depicted in figure 14 of the controller start-up strategy of Leithead et al. (1990a, b, 1991a, 1992) supports this connection to the anti-wind-up context for both the particular, essentially single-sided, position constraint in figure 14 and for more general position constraints. The discussion is confined to the issue of controller start-up and ignores, for the moment, the other implementation issues considered in this paper.

The approach of freezing the controller integral action during below rated operation is immediately seen to correspond to the conditional integration method, commonly described in the anti-wind-up literature (see, for example, Fertik \& Ross 1967, Astrom \& Rundquist 1987), of halting integral action when a hard limit is encountered and the control demand and its derivative have the same sign. The formulation of figure 14 subsumes the conditional integration approach which corresponds to the special case when the controller is partitioned such that the anti-wind-up feedback loop contains only a pure integrator and high (formally, infinite) gain. Whilst this simple approach works well for certain applications (Astrom \& Rundquist 1987), the performance can be poor, see figure 7, when the technique is applied to wind turbine control. The poor performance of the conditional integration approach in figure 7 is attributed to prolonged transients associated with the low frequency dynamics in the controller (Leithead et al. 1991a, 1992a). The improved performance of the approach of Leithead et al. (1990a, b, 1991a, 1992a) in figure 7 is associated with applying corrective feedback to both the pure integrator and the low frequency controller dynamics.

A further connection may be made with the high gain conventional anti-wind-up approach described by Kothare et al. (1994) (a fairly direct generalisation of the back-calculation approach (Fertik \& Ross 1967, Astrom \& Wittenmark 1984, Hanus et al. 1987)). However, this approach is not equivalent to that of Leithead et al. (1990a, b, 1991a, 1992a), since the formulation of Leithead et al. (1990a, b, 1991a, 1992a) employs dynamic feedback whilst the conventional anti-wind-up approach of Kothare et al. (1994) is expressly confined to static anti-wind-up feedback. Moreover, Leithead et al. (1990a, b, 1991a, 1992a) partition the controller such that only part of the dynamics of the controller are enclosed within the anti-wind-up loop whereas Kothare et al. (1994) apply high gain feedback around the entire controller. The performance of the high gain conventional anti-wind-up approach is not generally effective when applied to wind turbines. Figure 14 also reveals the similarity between the approach of Leithead et al. (1989, 1990a, b) and the model-based anti-wind-up approach independently proposed by Irving and briefly outlined by Hanus (1989) (but apparently not developed, beyond this initial suggestion, to the point of being practicable). However, it differs from the approach of Leithead et al. (1991a, 1992a) in several important respects, particularly with regard to the treatment of slow plant dynamics (see the discussion in section 3.2 concerning the dynamic inflow).

Campo et al. (1987) and Kothare et al. (1994) have proposed a rigorous synthesis approach, based on $\mu$-theory, for selecting anti-wind-up parameters. However, this approach is apt to be both rather 
conservative and relatively complex. Hence, it is often necessary, and indeed common practice, to exploit physical insight to minimise conservativeness and to develop simple heuristic tuning guidelines (Astrom \& Rundquist 1989 in the context of PI/PID control) such as those developed in section 5.2.

Whilst consideration is almost always restricted, in the general anti-wind-up context, to static compensation, the results discussed here indicate that, in appropriate circumstances, dynamic compensation can achieve superior anti-wind-up performance.

\section{Actuator Velocity/Acceleration Saturation}

It is well known that the hard limits present in all actuators can give rise to a degradation in control performance and may lead to a reduction in the stability margins and, indeed, instability. However, whilst it is noted in section 5 that a constraint on the output of the actuator can have a considerable impact on closed-loop performance, the effect on performance of velocity and acceleration constraints within the actuator is typically less marked since these constraints are normally only encountered for short periods of time. Velocity and acceleration constraints may, nevertheless, degrade the closed-loop stability margins. This is illustrated in figure 16 for the representative medium-scale turbine. Whilst the unconstrained system possesses a nominal gain margin of $10 \mathrm{~dB}$, the closed-loop system becomes unstable, exhibiting a large amplitude limit cycle, when actuator velocity constraints are introduced and the loop gain is increased by a factor of 2.5 ; that is, the actuator velocity constraints reduce the gain margin by $20 \%$. This type of behaviour is likely to be exacerbated in large-scale machines for which the increased size and inertia of the turbine rotor can require the actuator to be operated closer to its hard limits in order to meet the performance specification (Rogers \& Leithead 1994).

Although actuators are almost always subject to a combination of several constraints, the literature (with the notable exception of Fertik \& Ross 1967) is largely confined to the consideration of a simple position constraint on the output of the actuator. Moreover, the actuator dynamics are almost always neglected. Figure 16 indicates that the actuator limits on velocity and acceleration in wind turbines cannot be ignored implying, in turn, that the actuator dynamics cannot be neglected. Some simplification is possible, however, since it is almost always the case that only one of the velocity and acceleration constraints proves restrictive in practice and it is, therefore, sufficient to consider actuators with a single velocity or acceleration constraint. The discussion in this section is confined to a single velocity/acceleration constraint in the actuator.

The requirement is to devise a method to accommodate the presence of a velocity/acceleration constraint within the actuator. Other than requiring compatibility with the controller start-up arrangements discussed in section 5, the other implementation issues considered in this paper are ignored. A reset type of anti-wind-up formulation, whereby integral action is obtained via positive feedback (see, for example, Astrom \& Wittenmark 1984, Walgama \& Sternby 1990, Kothare et al. 1994), is unattractive owing to the compatibility requirements and the presence of significant actuator dynamics. Instead, the approach depicted in figure 17 directly extends the controller start-up/anti-windup approach, developed in section 5, to accommodate velocity/acceleration constraints (Rogers \& Leithead 1994, Leith \& Leithead 1995c). This formulation employs feedback of the difference, between the output of the real actuator, A, and a linear (unconstrained) model, $\hat{\mathrm{A}}_{u}$, to the input of the $\mathrm{C}_{\text {inner }}$ block in the controller via a suitable filter, F. A measurement of the control demand is always available within the controller and, when the actuator output is not measured, it may be estimated from an appropriate model of the actuator which includes a representation of the velocity/acceleration constraints (in a similar manner to the approaches proposed by Astrom \& Wittenmark 1984, Doyle et al. 1987, and others).

The stability analysis techniques discussed in section 5 may also be applied to provide sufficiency conditions for the stability of the controlled system when the foregoing velocity/acceleration constraint compensation approach is employed. Applying small gain analysis to the example medium-scale wind turbine, let $\mathrm{y}$ denote the input to the velocity saturation and $\mathrm{x}$ denote the output. In closed-loop operation, $\mathrm{y}$ is related to $\mathrm{x}$ by the transfer function

$$
y=\frac{\left(\mathrm{M}_{\mathrm{o}} \mathrm{C}_{\text {inner }} \mathrm{F} \hat{\mathrm{A}}_{\mathrm{u}}-\mathrm{M}_{\mathrm{o}} \mathrm{C}_{\text {inner }} \mathrm{C}_{\text {outer }} \mathrm{G}-\mathrm{C}_{\text {inner }} \mathrm{F} \hat{\hat{A}}_{\mathrm{u}} \mathrm{T}-\mathrm{T}\right) \mathrm{M}_{1} / \mathrm{s}}{1+\mathrm{C}_{\text {inner }} \mathrm{F} \hat{\mathrm{A}}_{\mathrm{u}}} \mathrm{x}
$$


and the system may, therefore, be re-formulated with this linear transfer function in the forward path and with feedback via the velocity saturation nonlinearity. When F is selected to be a gain of unity, the small gain theorem indicates that, with anti-wind-up, the closed-loop system is stable for any saturation both in nominal conditions and with the loop gain increased by a factor of 2.5 . A plot of the magnitude of (32), when the saturation element is replaced by a fixed gain of 0.6 , is shown in figure 18; the magnitude is less than 1.664 , indicating stability for the class of position nonlinearities with norm in the range $(-0.001,1.201)$ which, of course, encompasses the saturation nonlinearity. When the loop gain is increased by 3.15 , the system is indicated to be stable for the norm of the saturation nonlinearity lying in the range $(0.087,1.003)$; that is, for the input to the saturation nonlinearity less than 11.5 times the saturation limit (115.0 degrees/second). The velocity is extremely unlikely to exceed such a large value in practice and the system may be taken as stable for practical purposes with the required gain margin of $10 \mathrm{~dB}$.

\section{Integrated Controller Realisation}

In the preceding sections, the implementation issues (compensation of the nonlinear aerodynamics, automatic controller start-up and accommodation of actuator constraints) are considered separately and appropriate strategies for their resolution are developed. It remains to combine these strategies to achieve a controller realisation which caters for all of the implementation issues in an integrated manner. The analysis of section 4 is clearly valid when operating above rated with the actuator unsaturated. It follows immediately that, in these circumstances, the controller realisation should appropriately linearise the nonlinear aerodynamics in accordance with the results in section 4 . In contrast, during below rated operation and/or during actuator saturation the control loop is effectively broken and linearisation of the aerodynamics is, therefore, not required. Instead, the objective is to ensure that the transition to above rated/ unsaturated operation is, in some sense, smooth.

Whilst, of course, small gain methods and $\mu$-theory may be employed to rigorously analyse a system with multiple saturation nonlinearities, the very conservative nature of the results obtained often greatly limits their utility in practice. Owing to the manner of wind turbine operation, the constraints on the control demand and actuator velocity/acceleration are rarely encountered simultaneously. Consequently, they can be considered separately, assuming that the controlled system is sufficiently robust to accommodate this approximation. Simulation results, employing a well-validated methodology, confirm the validity of this separation for wind turbines (Leith \& Leithead 1995c, 1996b). This approach permits a useful compromise to be achieved between rigour and conservativeness.

The controller realisations developed by Leithead et al. (1990a, b, 1991a, 1992a) achieve smooth controller start-up by incorporating a minor feedback loop which, in some sense, mimics the plant dynamics and enables the controller to continue operating when below rated. This start-up strategy compensates for the controller nonlinear gain in a natural manner by incorporating a nonlinear gain equal to $\partial \mathrm{T} / \partial \mathrm{p}$, see figure 8 , in the minor feedback loop. However, whilst both the controller and the minor loop contain a gain, which varies nonlinearly in above rated operation, these gains are constant during below rated operation. It follows that, with this strategy, the control loop is effectively linear with respect to the aerodynamics during both above and below rated operation. Whilst Leithead et al. (1991a, 1992a) developed this controller start-up strategy for a specific approach to aerodynamic linearisation, it is easily seen to be compatible with all of the linearisation techniques developed in section 4.3. However, the linearisation approaches of figure 13 are judged to be unsuitable for wind turbines, see section 4.3, and attention is, therefore, confined to the direct linearisation strategies of figure 11 .

The anti-wind-up strategy, developed in section 6 to accommodate actuator velocity/acceleration saturation, is a fairly direct generalisation of the start-up strategy of Leithead et al. (1990a, b, 1991a, 1992a). It might be expected, therefore, that the strategy for accommodating the nonlinear aerodynamics of the plant, discussed above for controller start-up, might extend to this case. However, the controller nonlinear gain need not, now, be constant when the actuator is saturated and nonlinear analysis of the minor loop gain therefore becomes necessary. In the light of the discussion in section 4 , a nonlinear gain equal to $\partial \mathrm{T} / \partial \mathrm{p}$ (without associated integral action) is insufficient for modelling the nonlinear behaviour of the aerodynamics with respect to the pitch angle. It follows, therefore, that an appropriate solution, which is compatible with the linearisation methods of section 4.3 , is to incorporate the function, $\hat{\mathrm{h}}(\bullet)$, in the anti-wind-up feedback loop in order to compensate for the controller nonlinear gain. The controller start-up and anti-wind-up strategies may be combined immediately to 
give a single minor feedback loop which is active during below rated operation and when the actuator is saturated.

When the aerodynamic linearisation strategy of figure $11 \mathrm{~b}$ is employed, the foregoing considerations lead to the controller realisation depicted in figure 19. This realisation provides an integrated strategy for resolving the issues of nonlinear aerodynamics, controller start-up and actuator saturation. Its effectiveness has been confirmed by extensive simulation studies (Leith \& Leithead 1995c). When the actuator dynamics are sufficiently fast and the actuator saturation limits are such that they may be neglected, the realisation of figure 19 reduces, essentially, to that of Leithead et al. (1991a, 1992a). An equivalent realisation, based on the linearisation strategy of figure $11 \mathrm{a}$, is readily obtained.

The importance of adopting an appropriate controller realisation is illustrated by figure 20 for the medium-scale wind turbine. The appropriate controller realisation enables a substantial improvement in performance to be obtained in comparison to a poor controller realisation (with the nonlinear aerodynamic gain positioned after the pure integrator in the controller, conditional integration antiwind-up at start-up, no velocity anti-windup and consequently the requirement for a reduction in the controller gain in order to maintain adequate stability margins). The poor controller realisation is quite plausible and, indeed, its use has been observed on commercial wind turbines. Note that the example in figure 20 illustrates the performance for a moderate wind speed in order to emphasise the considerable importance of the controller realisation even under relatively benign wind conditions; at higher wind speeds the performance gains associated with an appropriate realisation become still more pronounced.

\section{Summary and Conclusions}

In this paper, three of the important, generic, implementation issues encountered when developing controllers for pitch-regulated constant-speed wind turbines are considered, namely

1. Compensation of the strongly nonlinear blade aerodynamics.

2. Automatic controller start-up/shut-down.

3. Accommodation of velocity and acceleration constraints within the actuator.

It is shown that the aerodynamic behaviour of the rotor possesses a local separability property whereby the dependence of the aerodynamic torque on the effective wind speed and the blade pitch angle may be explicitly de-coupled. Whilst this property is local to a neighbourhood of the locus of equilibrium operating points, the neighbourhood is sufficiently large to include the normal operating envelope. The local separability property enables several strategies to be rigorously established to accommodate the nonlinear aerodynamics both by direct linearisation and by feedback linearisation. Although nominally the same, the strategies differ with respect to many important practical issues, including the prior knowledge necessary, robustness, sensor requirements, degree of complexity (and, related to this, the scope for simplification in certain circumstances) and compatibility with controller start-up and anti-wind-up strategies. The existing strategy for accommodating the nonlinear aerodynamics, originally developed on the basis of physical insight, is rigorously derived but it is determined that this strategy is only valid when the actuator bandwidth is sufficiently large. Whilst this requirement is typically satisfied in medium-scale wind turbines, it may not be for large-scale machines. However, the analysis enables the existing strategy to be directly extended to cater for all machines.

With respect to the issue of automatic start-up/shut-down of the wind turbine controller, a rigorous stability analysis is presented for the first time and novel design guidelines are proposed which can significantly reduce the power transients at controller start-up (by over $50 \mathrm{~kW}$ for a machine rated at $300 \mathrm{~kW}$ ). It is noted that a close connection exists between the, apparently rather specific, requirement of ensuring appropriate automatic controller start-up/shut-down as the wind speed fluctuates and the general issue of anti-wind-up in the field of constrained control. This connection is exploited to observe that solutions, developed for the specific wind turbine problem, are, in general, directly applicable to a broad class of constrained control problems. Several aspects of one particular windturbine controller start-up strategy are noted to be novel in the anti-wind-up context and of quite general application. Whilst consideration in the general anti-wind-up context is almost always restricted to static compensation, the results presented here demonstrate that, in appropriate circumstances, dynamic compensation can achieve superior anti-wind-up performance.

Whilst it is well known, particularly in the context of process control, that hard actuator limits can degrade performance and reduce the stability margins or, indeed, induce instability, this issue has previously been neglected in wind turbine applications. Typically, an actuator velocity constraint can reduce the gain margin of a pitch-regulated wind turbine by around $20 \%$. If adequate stability margins 
are not achieved a wind turbine system must sometimes destabilise, although not necessarily become unstable, in which case the wind turbine would experience large load fluctuations. The reduction in the stability margins is, furthermore, likely to be exacerbated on large-scale machines where, owing to the increased size and inertia of the turbine blades, the actuator may require to be operated at its hard limits to a greater extent in order to meet the performance specification. Since actuator constraints are always present, it follows that it is necessary, unless alternative corrective measures are employed, to de-tune the controller in comparison to the linear unconstrained situation in order to achieve adequate stability margins. Strategies for recovering adequate stability margins without de-tuning the controller are described in the anti-wind-up literature. However, these are rather limited in scope and are not directly applicable, in general, to wind turbines (and many other application areas) for which restrictive position, velocity and acceleration constraints may all be present and the dynamic behaviour of the actuator cannot be neglected. A novel, and quite general, anti-wind-up strategy is proposed which caters for these circumstances.

The separate strategies for resolving the implementation issues are combined to achieve an elegant controller realisation which accommodates all the implementation issues in an integrated manner. The importance of adopting an appropriate controller realisation is considerable and is illustrated for a 300 $\mathrm{kW}$ wind turbine.

The implementation issues considered in this paper are, of course, not confined to wind turbines but are of wider concern..

\section{Acknowledgements}

The support of the EPSRC, DTI (formerly D.En) and ETSU, by whose permission this paper is published, is gratefully acknowledged..

\section{References}

ASTROM, K.J., WITTENMARK, B., 1984, Computer Controlled Systems: Theory \& Design (Englewood Cliffs, N.J.: Prentice-Hall).

ASTROM, K.J., RUNDQWIST, L., 1989, Integrator Windup and How to Avoid It. Proceedings of the American Control Conference, 1693-1698.

ATHERTON, DP, 1982, Nonlinear Control Engineering (New York: Van Nostrand Reinhold).

BUCKLEY, P.S., 1971, Designing Override and Feedforward Controls - I \& II. Control Engineering, 18, 48-51 \& 82-85.

CAMPO, P.J., MORARI, M., NETT, C., 1989, Multivariable Anti-Windup \& Bumpless Transfer: A General Theory. Proceedings of the American Control Conference, Pittsburgh, 1706-1711.

CAMPO, P.J., MORARI, M., 1990, Robust Control of Processes Subject to Saturation Nonlinearities. Computers Chem. Eng., 14, 343-358.

DESOER, C.A., VIDYASAGAR, M.,1975, Feedback Systems: Input-Output Properties (London : Academic Press).

DOYLE, J.C., SMITH, R.S., ENNS,S.F., 1987, Control of Plants with Input Saturation Nonlinearities. Proceedings of the American Control Conference,1034-1039.

FERTIK, H.A., ROSS, C.W., 1967, Direct Digital Control Algorithm with Anti-Windup Feature. ISA Transactions, 6, 317-328.

FRANKENA, J.F., SIVAN,R., 1979, A Nonlinear Optimal Control Law for Linear Systems. International Journal of Control, 30, 159-178.

GARCIA, C.E., PRETT, D.M., MORARI, M., 1989, Model Predictive Control: Theory and Practice. Automatica, 25, 335-348.,

GUTMAN, P., HAGANDER, P., 1985, A New Design of Constrained Controllers for Linear Systems. IEEE Transactions on Automatic Control, 30, 22-33.

HANUS, R., 1989, Anti-windup and Bumpless Transfer: A Survey. Computing \& Computers for Control Systems (Ed. Borne, P.), $12^{\text {th }}$ IMACS World Congress on Scientific Computation, Paris..

HANUS, R., KINNAERT, M., HENROTTE, J.L., 1987, Conditioning Technique, a General Antiwindup and Bumpless Transfer Method. Automatica, 23, 729-739.

HEISE, S.A., MACIEJOWSKI, J.M., 1994, Stability of Constrained MBPC using an internal model control structure. pp230-244 in Advances in Model-Based Predictive Control edited by D.Clarke (New York: Oxford University Press).

ISIDORI, A., 1989, Nonlinear Control Systems: An Introduction (Springer Verlag). 
KHALIL, H.K., KOKOTOVIC, P.V., 1991, On Stability Properties of Nonlinear Systems with Slowly Varying Inputs. IEEE Transactions on Automatic Control, 36, 229.

KOTHARE, M.V., CAMPO, P.J., MORARI, M., NETT, C.N., 1994, A Unified Framework for the Study of Antiwindup Designs. Automatica, 30, 1869-1883.

LEITH, D.J., LEITHEAD, W.E., 1995a, Directly Responding to Peak Power Excursions in PitchRegulated HAWTs. Proceedings of the British Wind Energy Association Conference.

LEITH, D.J., LEITHEAD, W.E., 1995b, Strongly Input Constrained Nonlinear Control of a Horizontal Axis Wind Turbine. Proceedings of the European Control Conference, 1562-1567.

LEITH, D.J., LEITHEAD, W.E., 1995c, An Investigation of the Benefits of Nonlinear Control for Pitch-Regulated Wind Turbines. Industrial Control Centre, University of Strathclyde report prepared under DTI contract no. ETSU W/42/00348/00/00.

LEITH, D.J., LEITHEAD, W.E., 1996a, Appropriate Realisation of Gain-Scheduled Controllers with Application to Wind Turbine regulation. To be published in International Journal of Control.

LEITH, D.J., LEITHEAD, W.E., 1996b, Performance Enhancement of Wind Turbine Power Regulation by Switched Linear Control. To be published in International Journal of Control.

LEITHEAD, W.E., AGIUS, P., 1991, Application of Classical Control to the WEG MS3 Wind Turbine. Report ICU/342, Industrial Control Centre, Department of Electronic \& Electrical Engineering, University of Strathclyde, Glasgow.

LEITHEAD, W.E., DE LA SALLE, S.A., REARDON, D.L., GRIMBLE, M.J., 1989, Wind Turbine Modelling and Control. Report prepared for the Department of Energy, May 1989.

LEITHEAD, W.E., DE LA SALLE, S.A., REARDON, D.L., 1990a, Classical Control of a Pitch Control System. Proceedings of the British Wind Energy Association Conference, Norwich.

LEITHEAD, W.E., DE LA SALLE, S.A., REARDON, D.L., 1990b, The Application of Classical Control to a Wind Turbine. Report prepared for the Department of Energy, 1990.

LEITHEAD, W.E., DE LA SALLE, S.A., REARDON, D.L., GRIMBLE, M.J., 1991a, Wind Turbine Control Systems Modelling and Design Phase I and II. Department of Trade \& Industry Report No. ETSU WN 5108.

LEITHEAD, W.E., DE LA SALLE, S.A., REARDON, D., 1991b, Role and Objectives of Control for Wind Turbines. Proceedings of the Institution of Electrical Engineers Pt C, 138, 135-148.

LEITHEAD,W.E., DE LA SALlE, S.A., REARDON, D., 1992a, Classical Control of Active Pitch Regulation of Constant Speed HAWTs. International Journal of Control, 55, 845-876.

LEITHEAD, W.E., ROGERS, M.C.M., AGIUS, P.R.D., 1992b, Dynamic Analysis of the Compliant Tip. University of Strathclyde report prepared for the Department of Energy (ETSU).

LEITHEAD, W.E., ROGERS, M.C.M., LEITH, D.J., CONNOR, B., 1995, Design of Wind Turbine Controllers. Proceedings of EURACO Workshop 'Recent Results in Robust \& Adaptive Control', Florence.

ROGERS, M.C.M., LEITHEAD, W.E., 1994, Development of Control System for RES 1 MW Machine. Industrial Control Centre, University of Strathclyde report prepared for Renewable Energy Systems Ltd.

SHAMMA, J.S., ATHANS, M., 1990, Analysis of Gain Scheduled Control for Nonlinear Plants. IEEE Transactions on Automatic Control, 35, 898-907.

SHINSKEY, F.G., 1967 \& 1988, Process Control Systems (New York: McGraw-Hill).

SLOTINE, ,J.E., LI, W., 1991, Applied Nonlinear Control (Englewood Cliffs, N.J.: Prentice-Hall).

SUSSMAN, H.J., SONTAG, E.D., YANG, Y., 1994, A General Result on the Stabilization of Linear Systems Using Bounded Controls. IEEE Transactions on Automatic Control, 39, 2411-2425.

WALGAMA, K.S., STERNBY, J., 1990, Inherent Observer Property in a Class of Anti-windup Compensators. International Journal of Control, 52, 705-724.

WREDENHAGEN, G., BELANGER, P., 1994, Piece-wise Linear LQ Control for Systems with Input Constraints. Automatica, 30, 403-416. 
Figure 1 Block diagram representation of wind turbine control loop

Figure 2a Dynamic relationship of aerodynamic torque to pitch angle and effective wind speed

Figure 2b Local linearisation of aerodynamic non-linearity.

Figure 2c Block diagram decomposition of aerodynamic non-linearity

Figure 3 Actuator configuration

Figure 4 Bode plot of open-loop transfer function of $300 \mathrm{~kW}$ machine

Figure 5a Block diagram representation of aerodynamic compensation approach of Leithead et al. (1990a, b, 1991a).

Figure 5b Block diagram representation of gain-scheduling aerodynamic compensation.

Figure 6 Typical power time histories for $300 \mathrm{~kW}$ machine at $16 \mathrm{~m} / \mathrm{s}$ mean wind speed with nonlinear gain positioned before $\&$ after pure integrator in controller ${ }^{2}$

Figure 7 Typical below to above rated transition for $300 \mathrm{~kW}$ machine ${ }^{2}$

Figure 8 Controller start-up approach of Leithead et al. (1990a, b, 1991a)

Figure 9 Strategy of Leithead et al. (1991a, 1992a) to resolve the implementation issues

Figure 10 Plot of aerodynamic torque point values vs. $\varepsilon$ illustrating the range over which a function $\tau(\varepsilon)$ provides an accurate representation.

Figure 11a Direct linearisation

Figure 11b Direct linearisation with velocity form

Figure 11c Reformulation of figure $11 b$ for robustness analysis.

Figure 12 Typical power time histories for $1 \mathrm{MW}$ machine at $12 \mathrm{~m} / \mathrm{s}$ mean wind speed with \& without compensation for the actuator dynamics ${ }^{2}$.

Figure 13a Conventional feedback linearisation.

Figure 13b Velocity form of feedback linearisation.

Figure 14 Reformulation of controller start-up approach of Leithead et al. (1990a, b, 1991a, 1992a)

Figure 15 Plot of $\left|\mathrm{C}_{\text {inner }}\left(\mathrm{F}_{-\mathrm{GA}}\right) /\left(1+\mathrm{FC}_{\text {inner }}\right)\right|$ for $300 \mathrm{~kW}$ wind turbine

Figure 16 Limit cycle induced by velocity constraints without anti-wind-up and corresponding stable response when anti-wind-up is employed ${ }^{2}$.

Figure 17 Anti-wind-up arrangement for velocity/acceleration saturation magnitude

Figure 18 Small gain analysis of closed-loop system with loop gain increased by a factor of 2.5.

Figure 19 Controller realisation which addresses the implementation issues in an integrated manner ${ }^{1}$.

Figure 20 Impact of controller implementation on power output of medium-scale example machine (16 $\mathrm{m} / \mathrm{s}$ mean wind speed, $20 \%$ turbulence intensity).

Table 1 Aerodynamic coefficients for medium-scale wind turbine

\footnotetext{
2 except for the specific details noted in the text the integrated realisation discussed in section 7 was employed.
} 


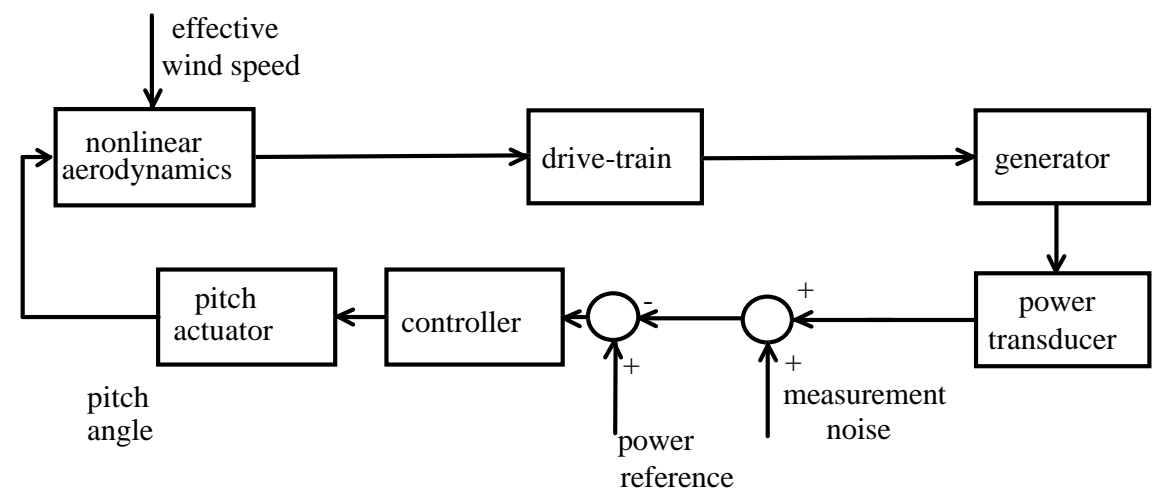

Figure 1 Block diagram representation of wind turbine control loop

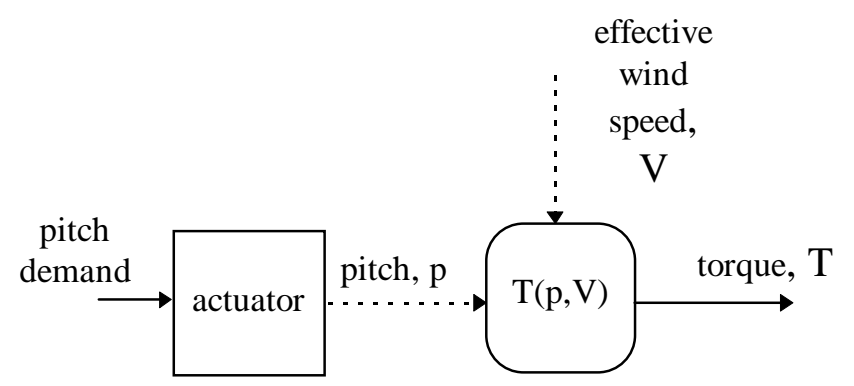

Figure 2a Dynamic relationship of aerodynamic torque to pitch angle and effective wind speed

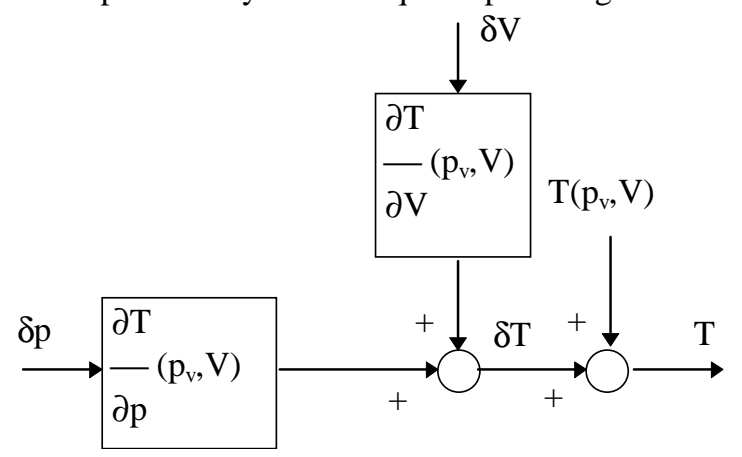

Figure 2b Local linearisation of aerodynamic non-linearity.

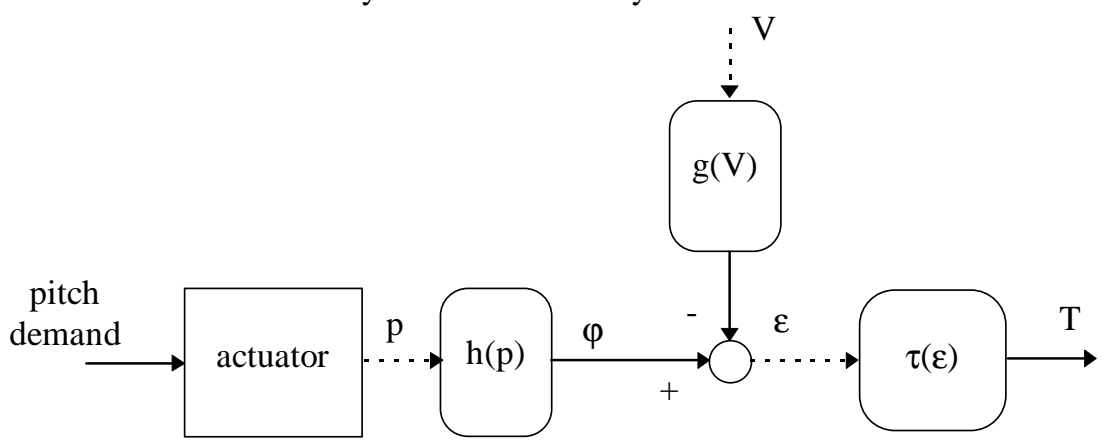

Figure 2c Block diagram decomposition of aerodynamic non-linearity

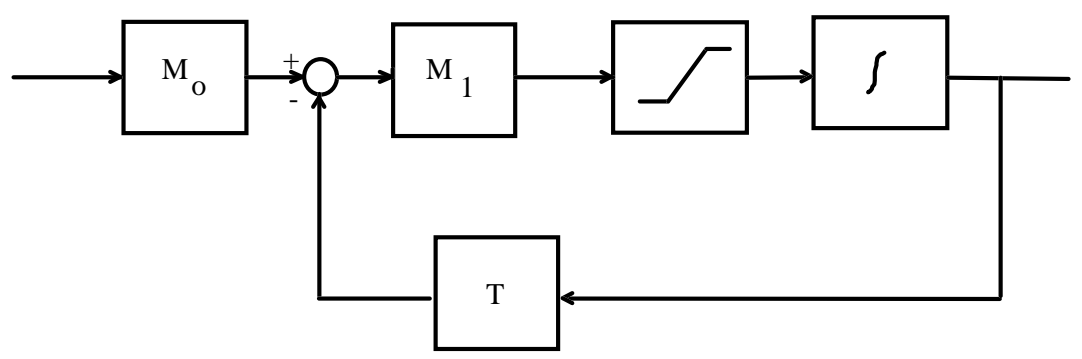

Figure 3 Actuator configuration 

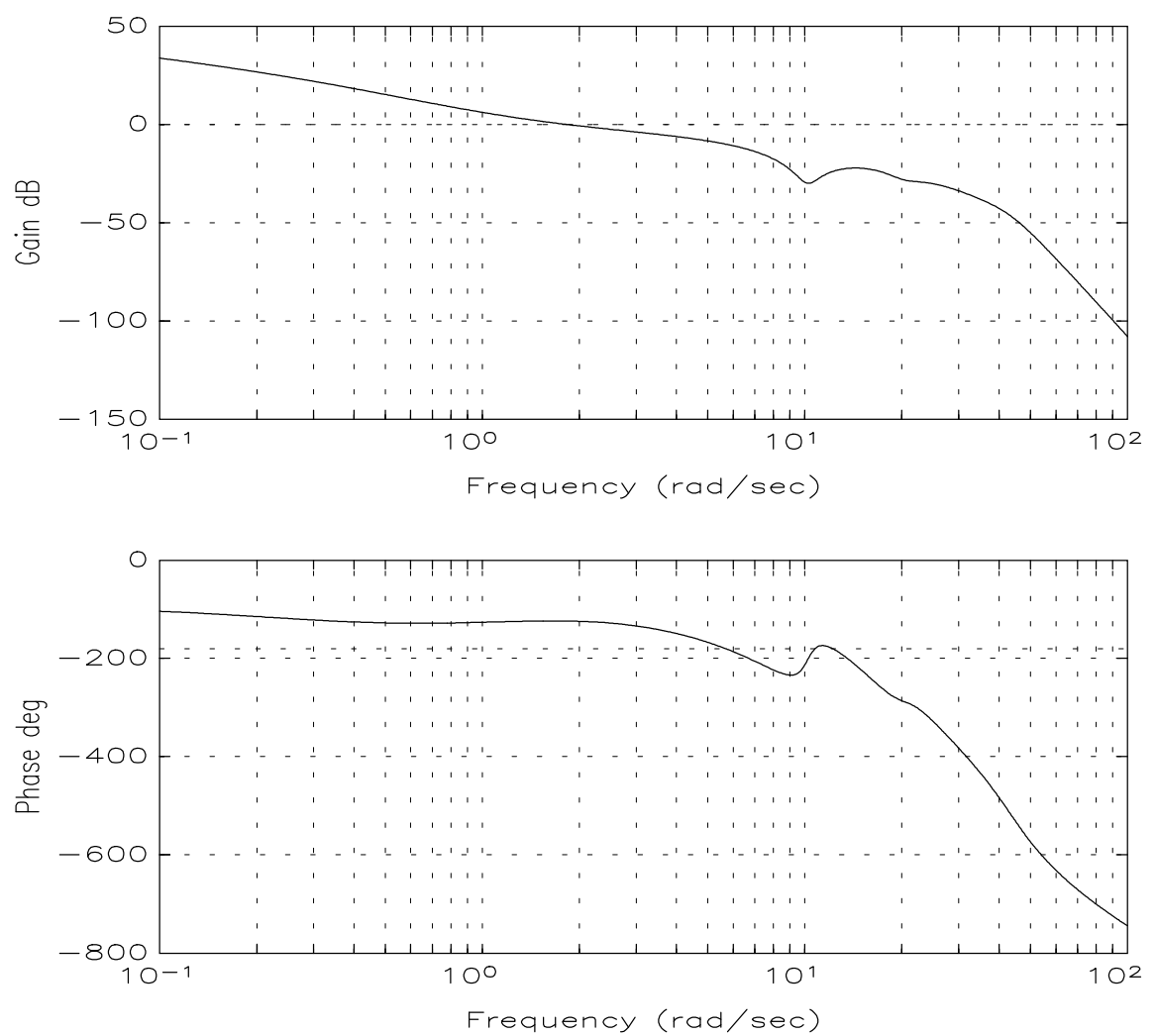

Figure 4 Bode plot of open-loop transfer function of $300 \mathrm{~kW}$ machine

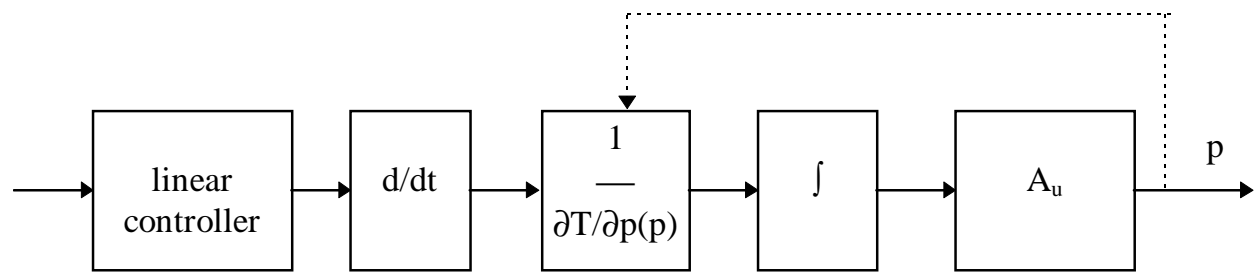

Figure 5a Block diagram representation of aerodynamic compensation approach of Leithead et al. (1990a, b, 1991a).

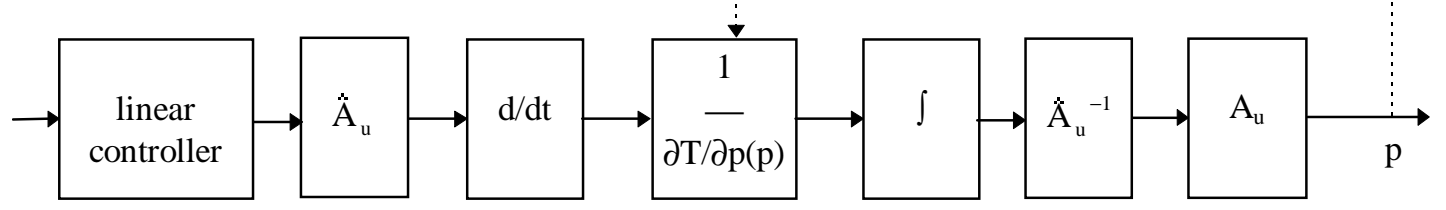

Figure 5b Block diagram representation of gain-scheduling aerodynamic compensation. 


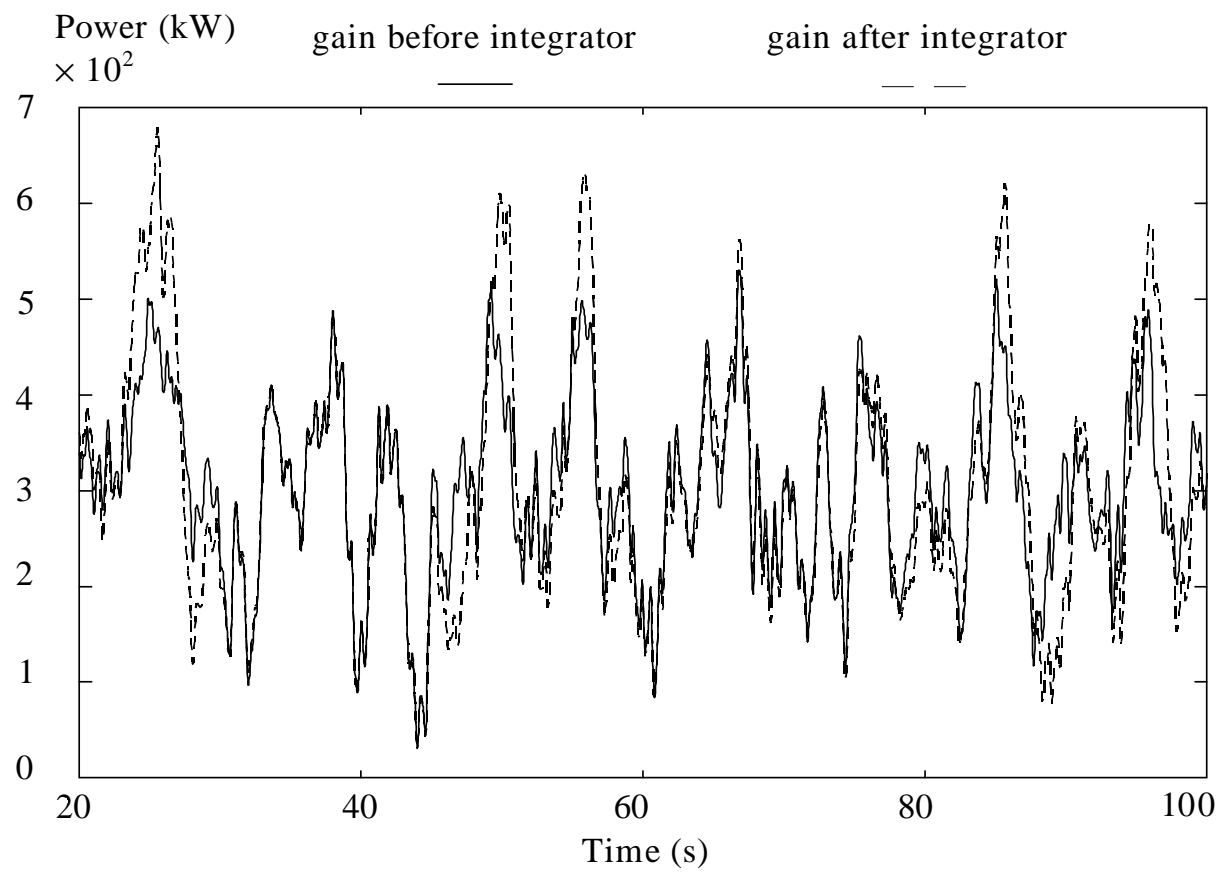

Figure 6 Typical power time histories for $300 \mathrm{~kW}$ machine at $16 \mathrm{~m} / \mathrm{s}$ mean wind speed with nonlinear gain positioned before $\&$ after pure integrator in controller ${ }^{2}$

power (W)

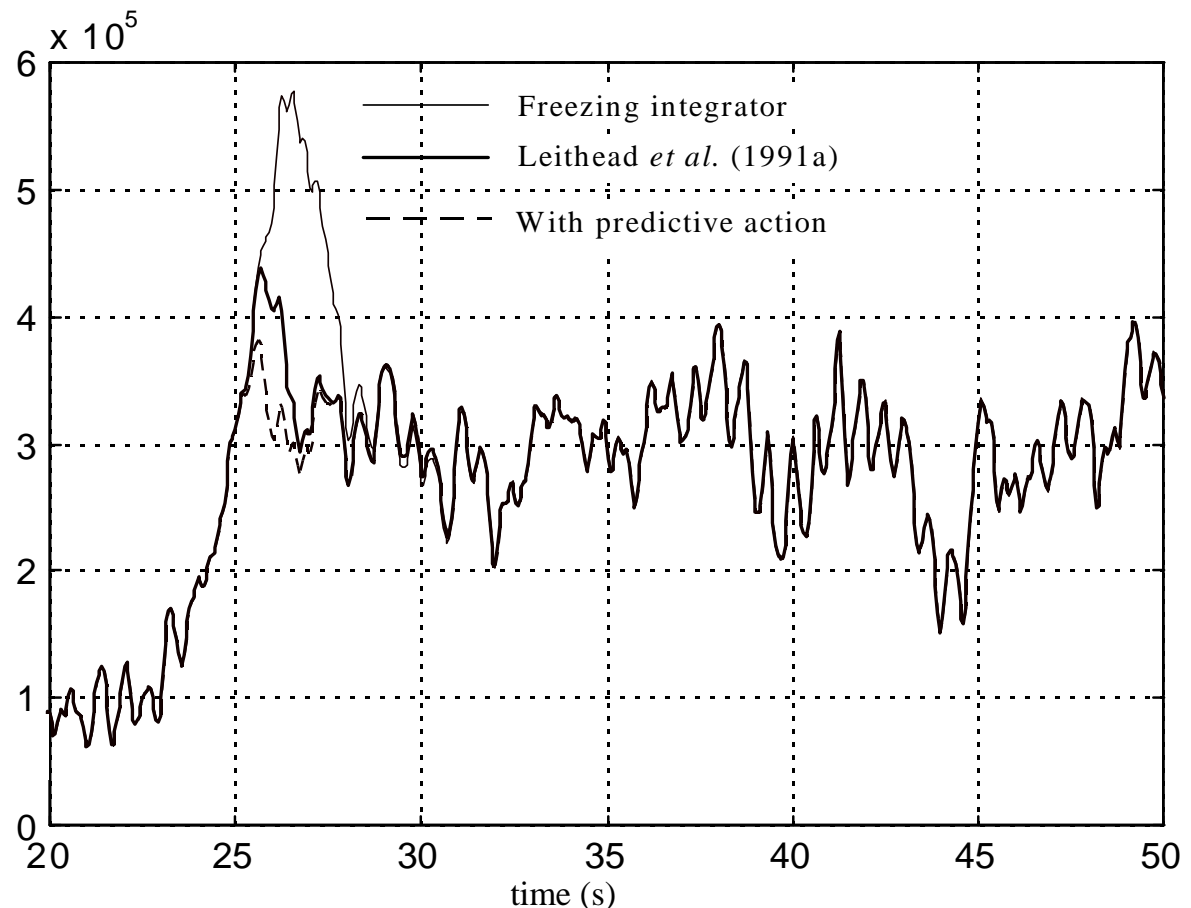

Figure 7 Typical below to above rated transition for $300 \mathrm{~kW}$ machine ${ }^{2}$

\footnotetext{
2 except for the specific details noted in the text the integrated realisation discussed in section 7 was employed.
} 


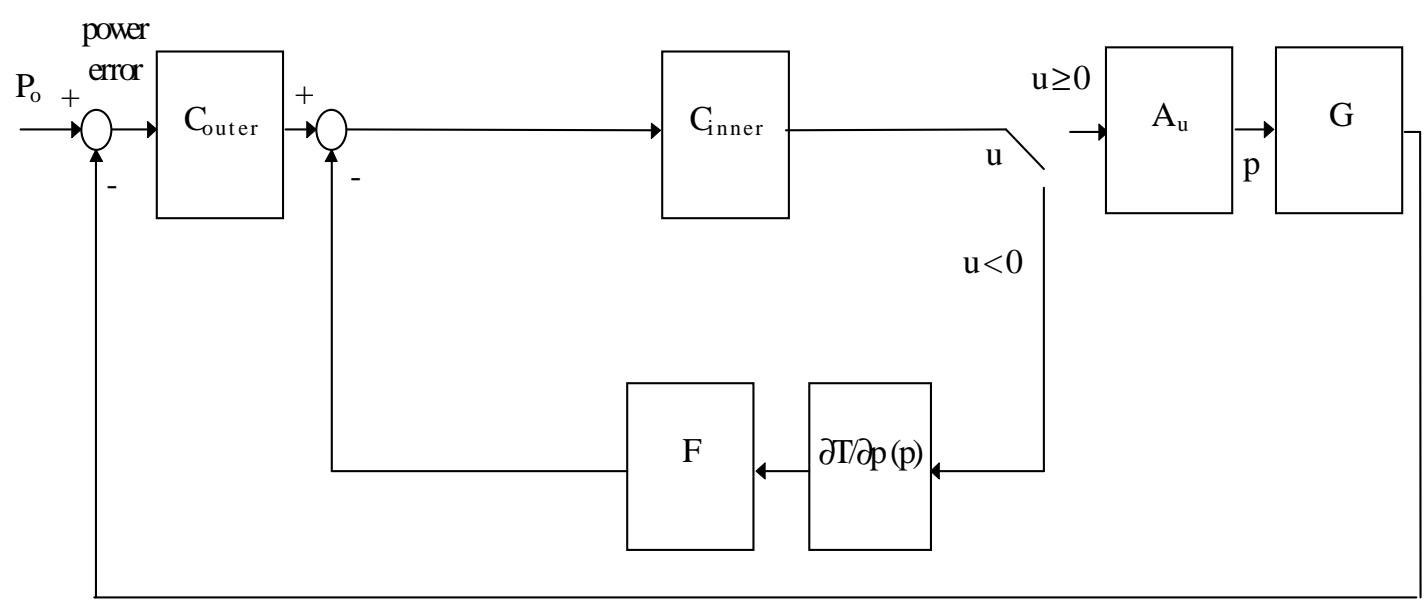

Figure 8 Controller start-up approach of Leithead et al. (1990a, b, 1991a)

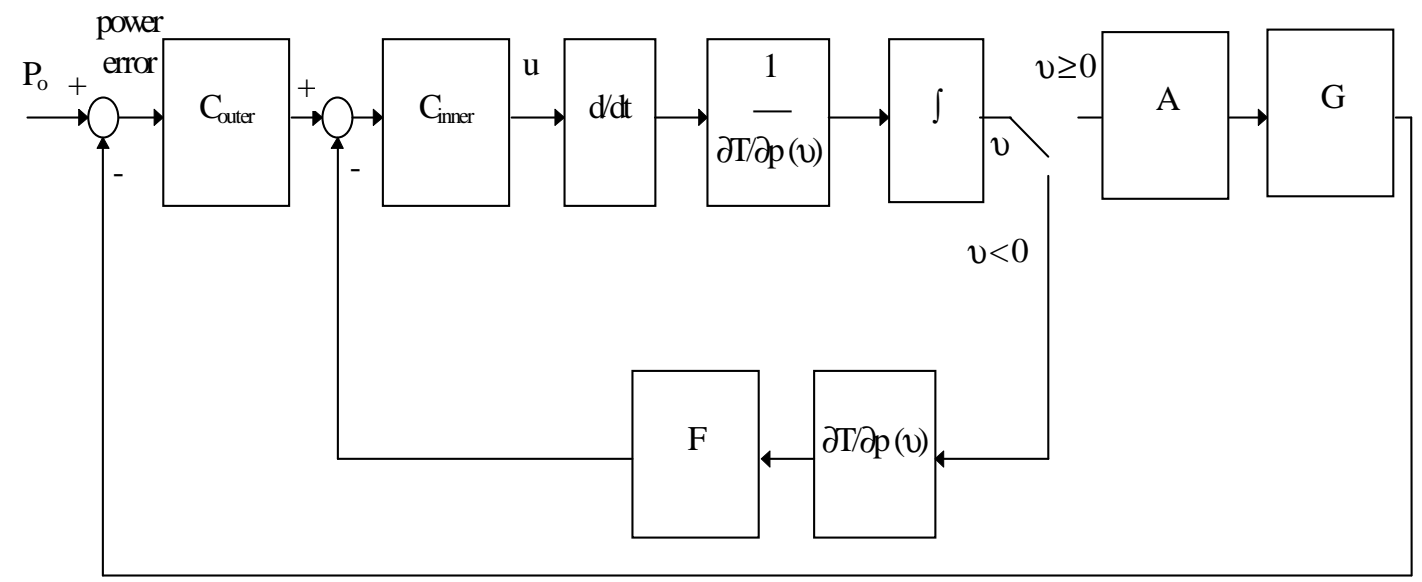

Figure 9 Strategy of Leithead et al. (1991a, 1992a) to resolve the implementation issues

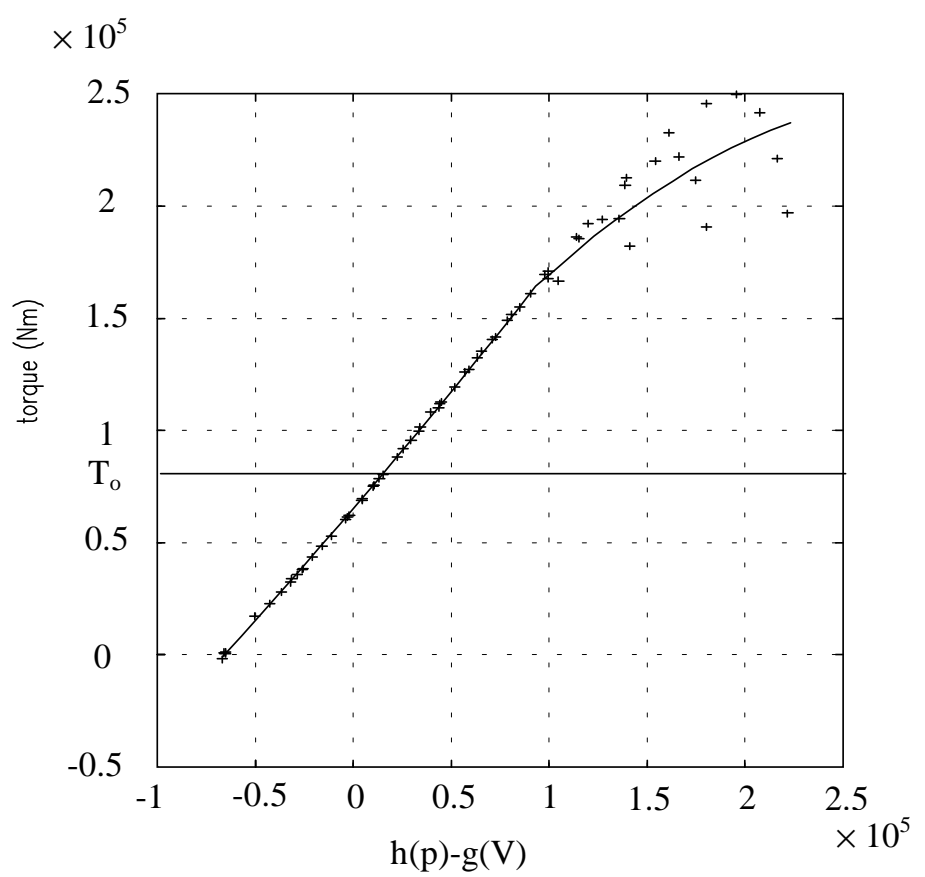

Figure 10 Plot of aerodynamic torque point values vs. $\varepsilon$ illustrating the range over which a function $\tau(\varepsilon)$ provides an accurate representation. 


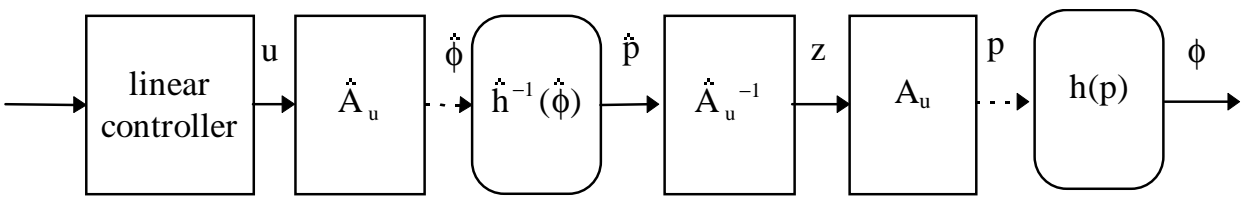

Figure 11a Direct linearisation

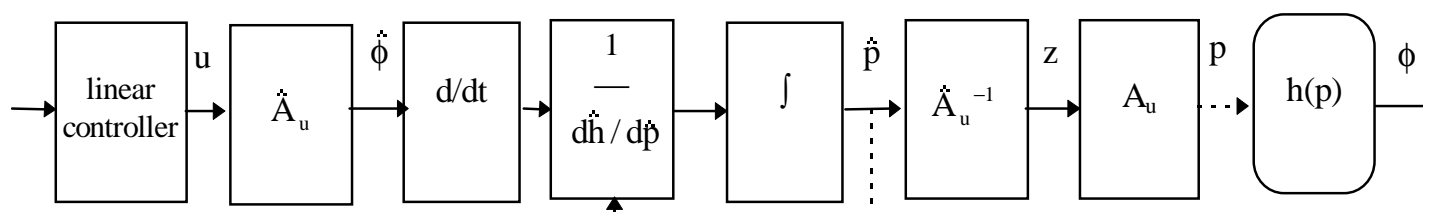

Figure 11b Direct linearisation with velocity form

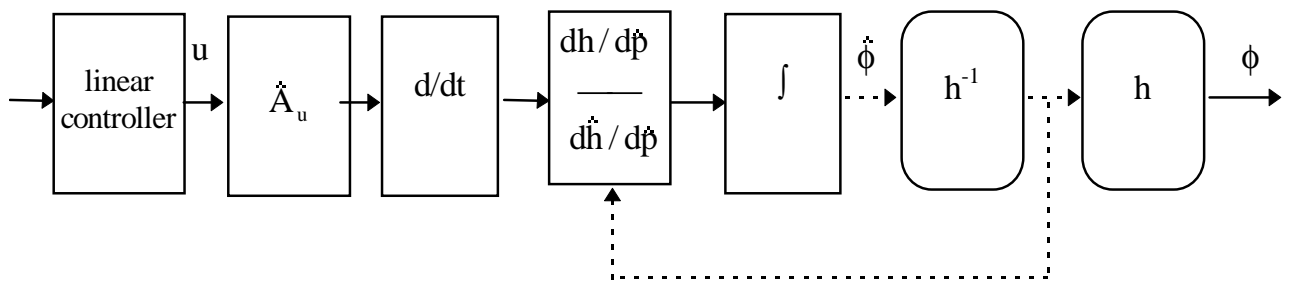

Figure 11c Reformulation of figure $11 \mathrm{~b}$ for robustness analysis.

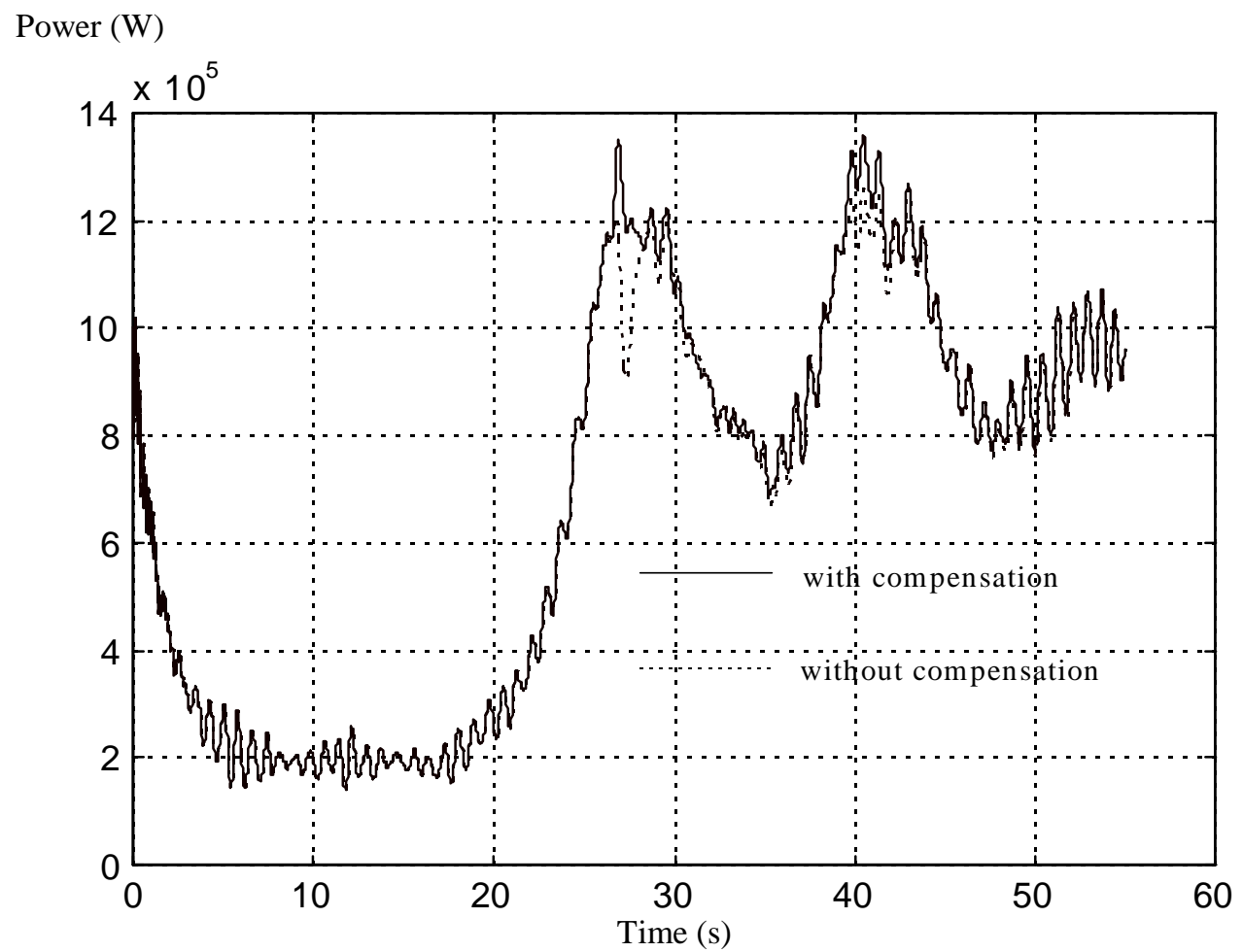

Figure 12 Typical power time histories for $1 \mathrm{MW}$ machine at $12 \mathrm{~m} / \mathrm{s}$ mean wind speed with \& without compensation for the actuator dynamics ${ }^{2}$. 


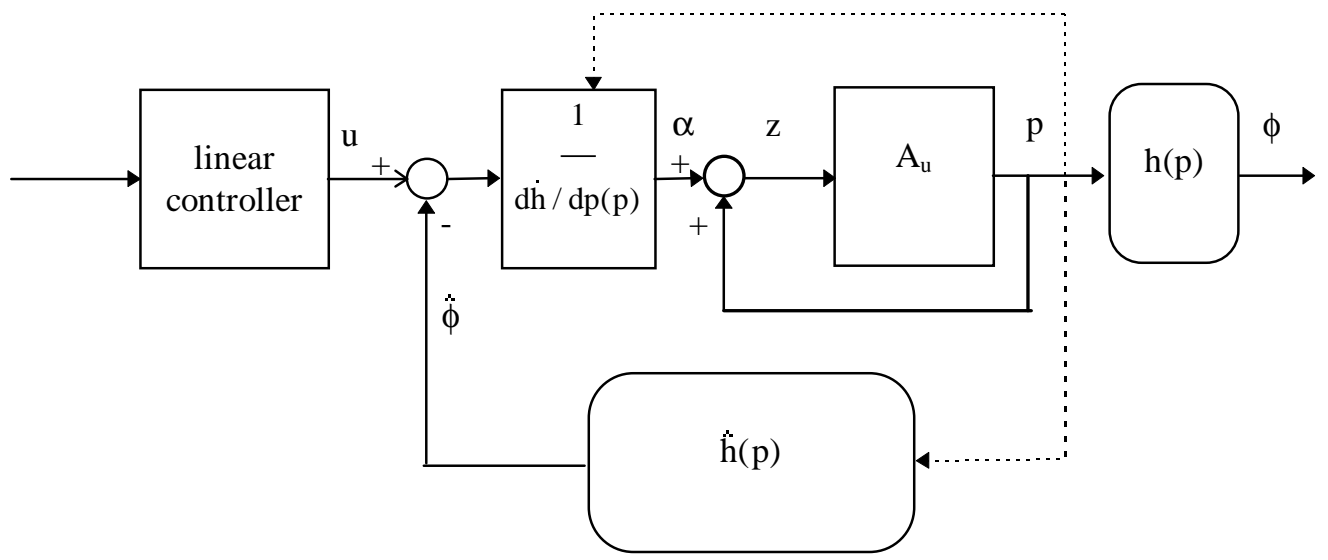

Figure 13a Conventional feedback linearisation.

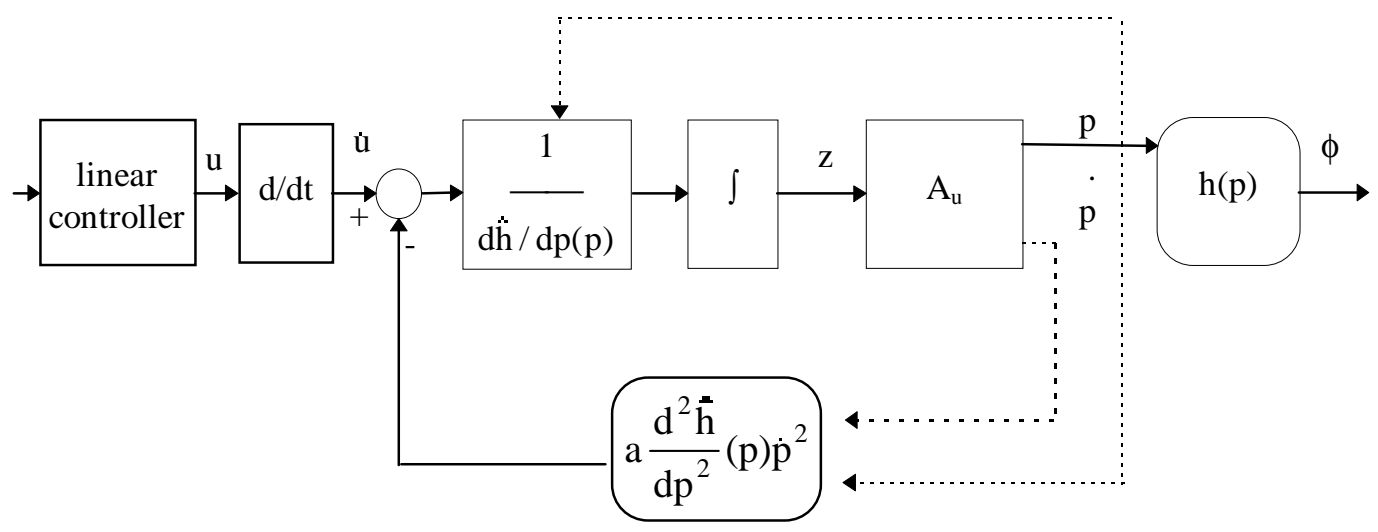

Figure 13b Velocity form of feedback linearisation.

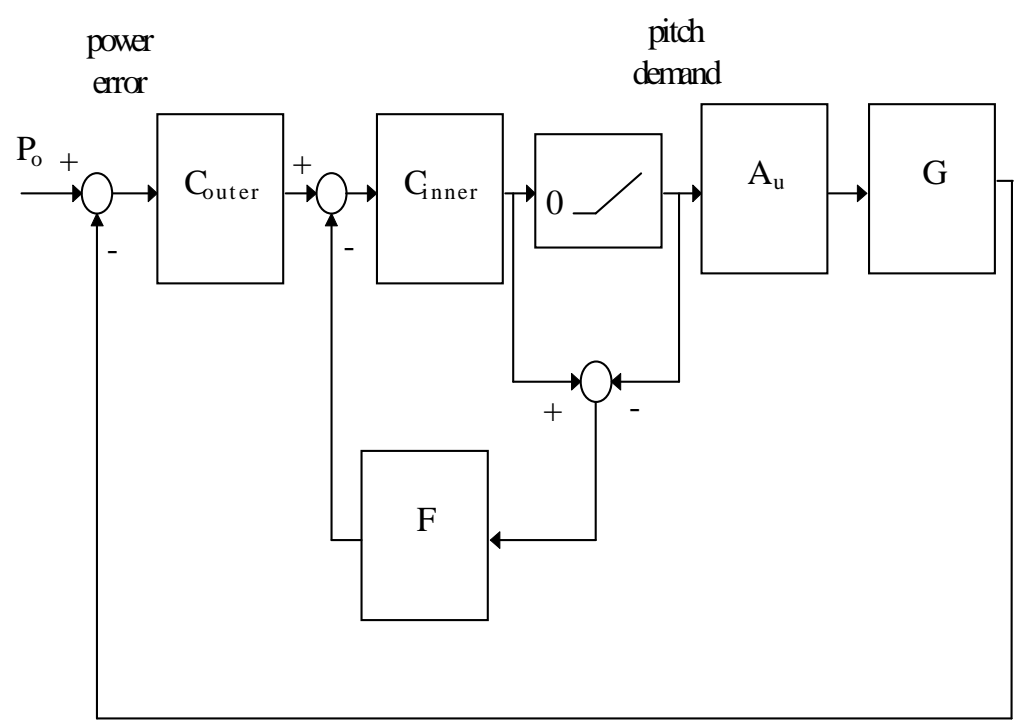

Figure 14 Reformulation of controller start-up approach of Leithead et al. (1990a, b, 1991a, 1992a) 


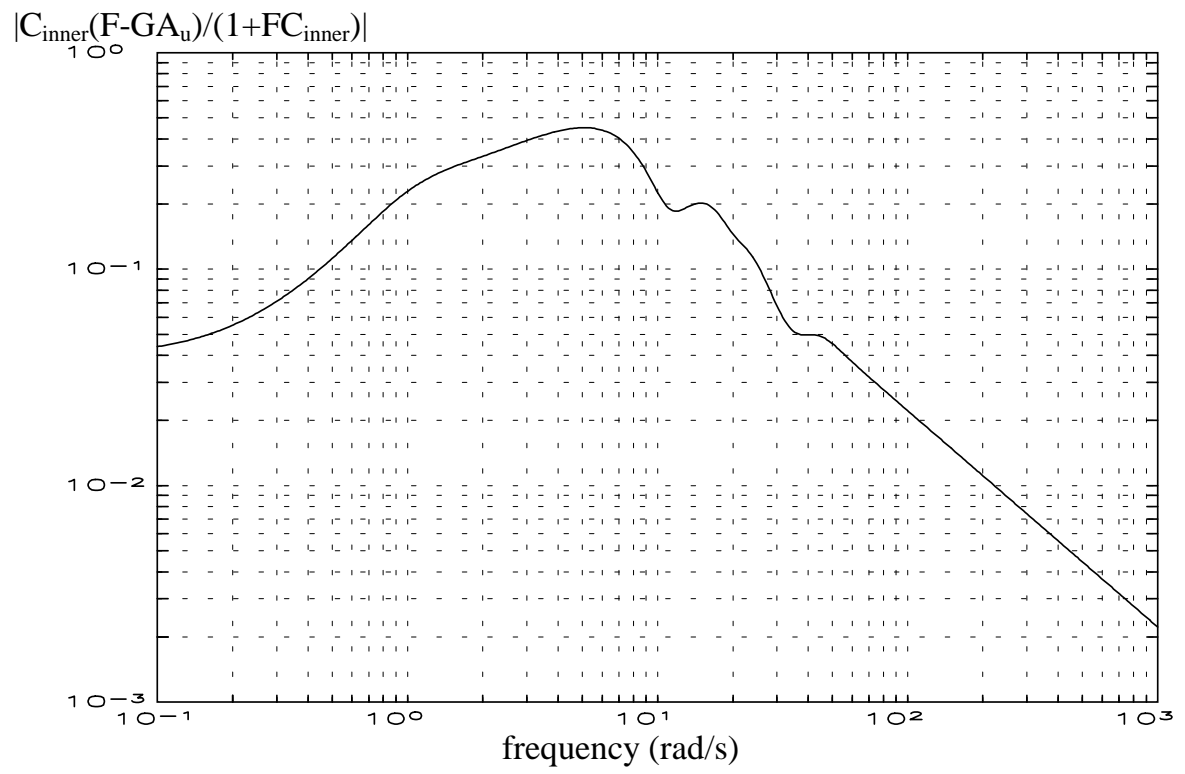

Figure 15 Plot of $\left|\mathrm{C}_{\text {inner }}\left(\mathrm{F}_{-\mathrm{GA}}\right) /\left(1+\mathrm{FC}_{\mathrm{inner}}\right)\right|$ for $300 \mathrm{~kW}$ wind turbine

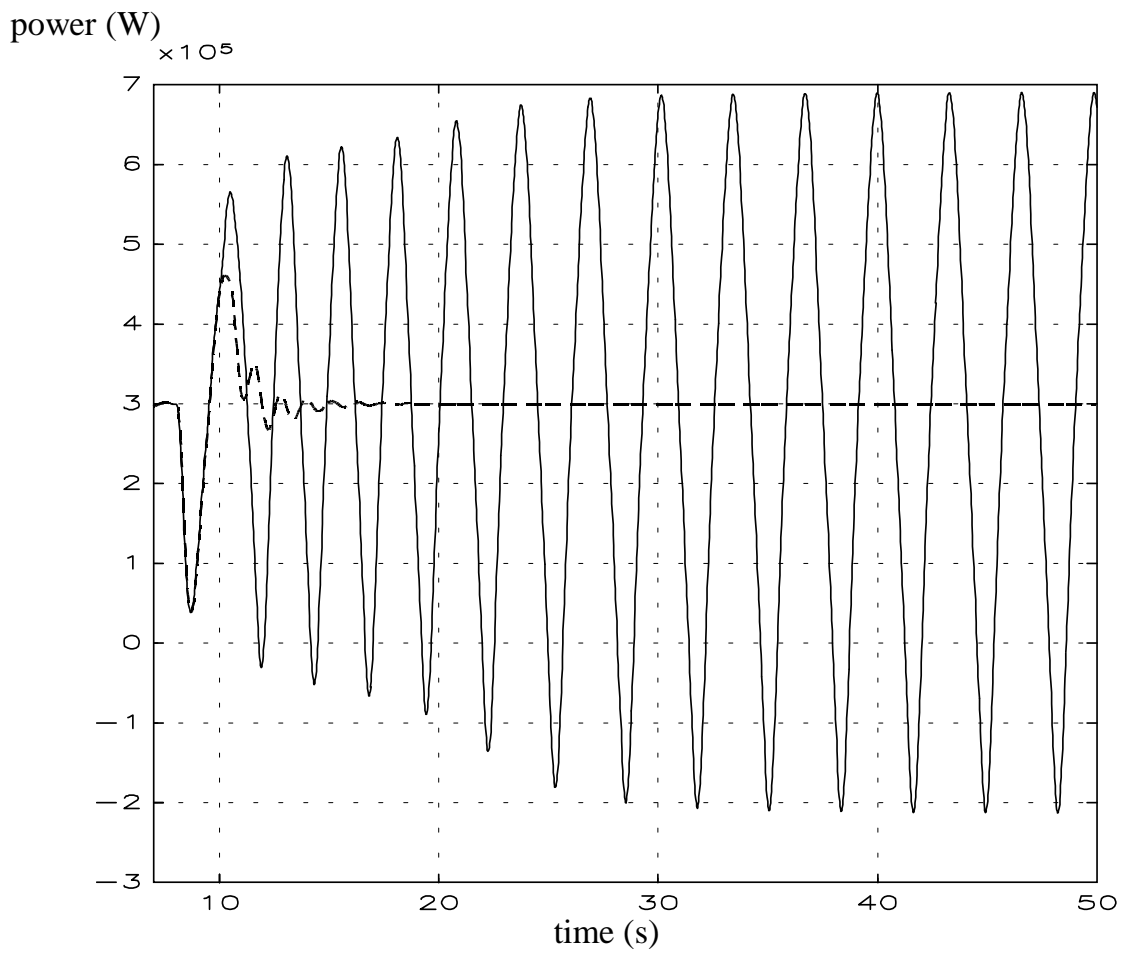

Figure 16 Limit cycle induced by velocity constraints without anti-wind-up and corresponding stable response when anti-wind-up is employed ${ }^{2}$.

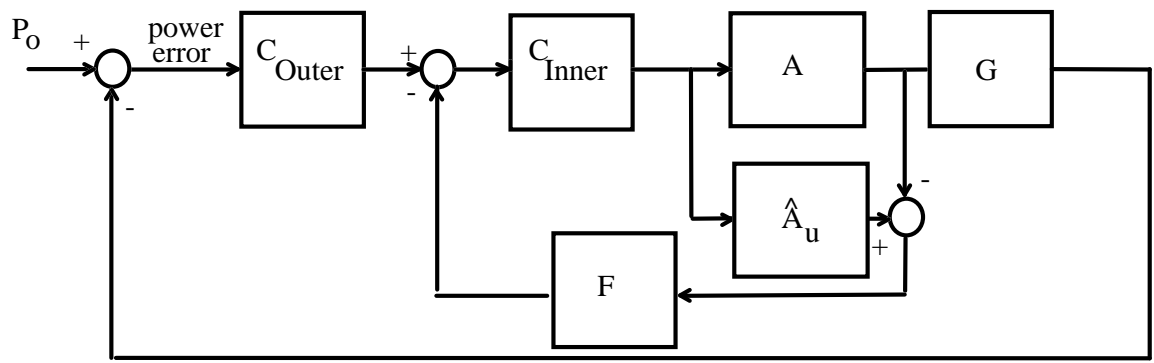

Figure 17 Anti-wind-up arrangement for velocity/acceleration saturation magnitude 


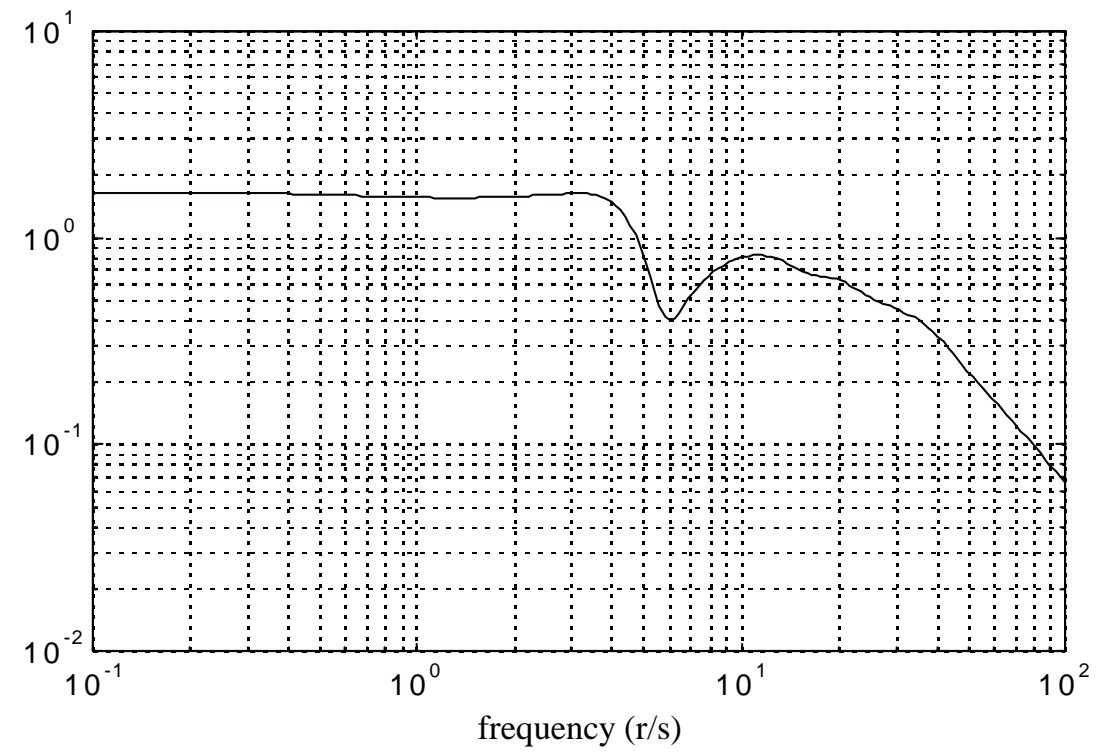

Figure 18 Small gain analysis of closed-loop system with loop gain increased by a factor of 2.5.

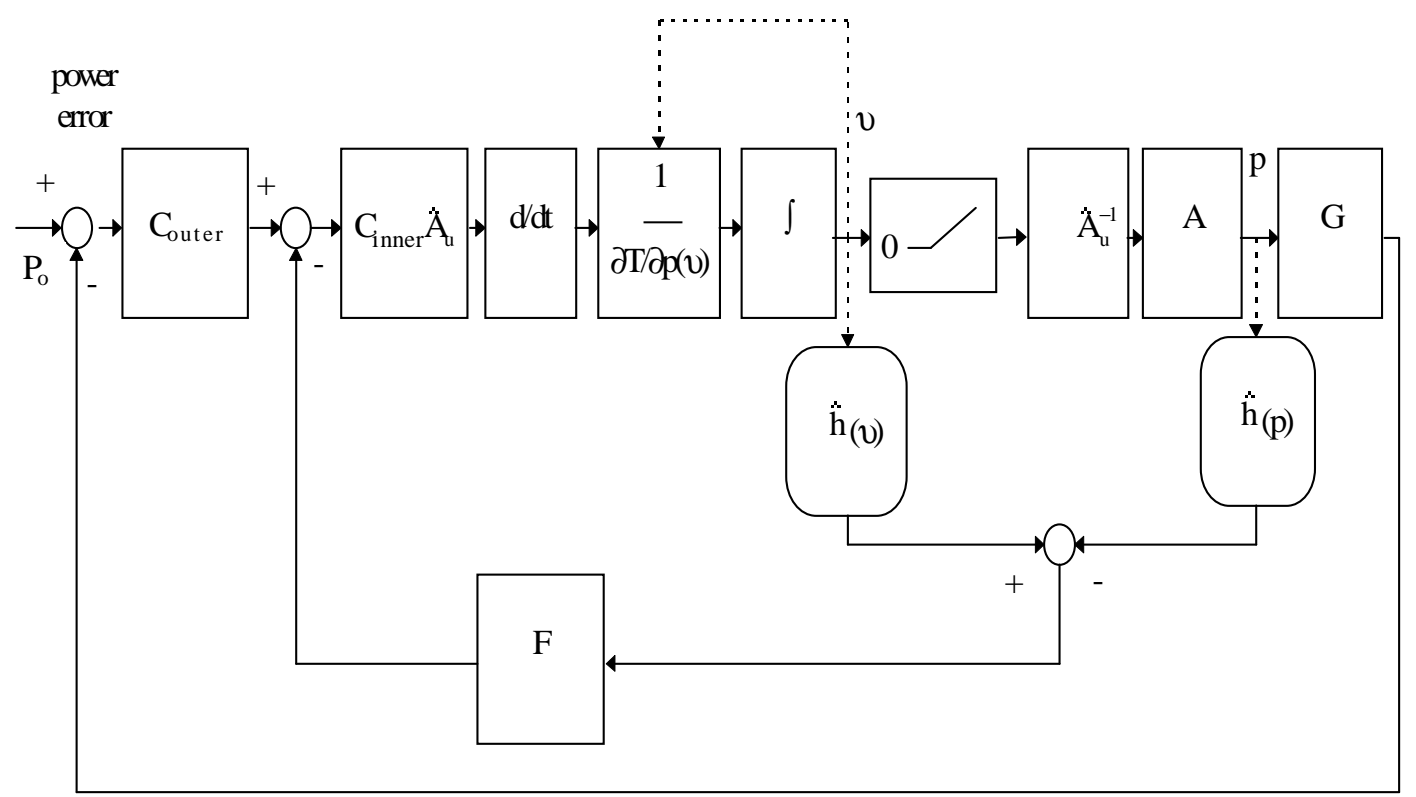

Figure 19 Controller realisation which addresses the implementation issues in an integrated manner ${ }^{1}$. 
Power (W)

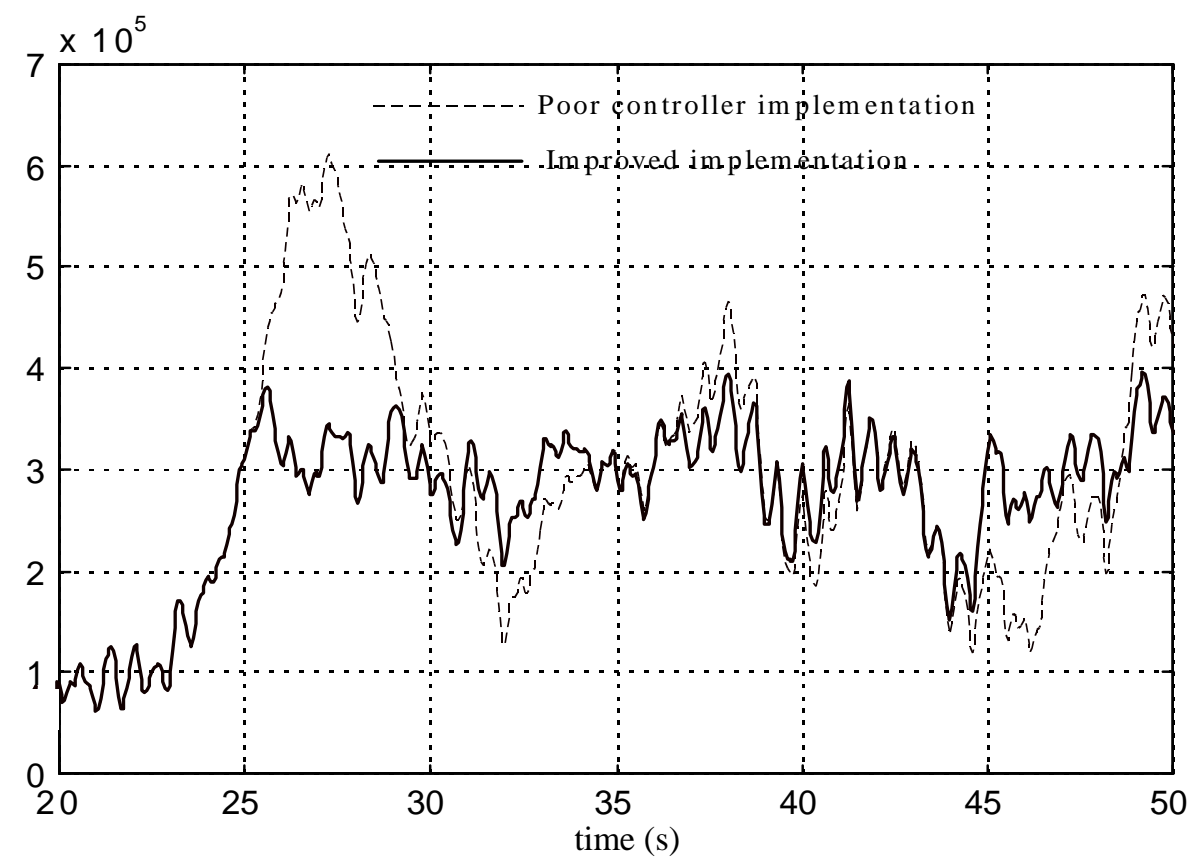

Figure 20 Impact of controller implementation on power output of medium-scale example machine (16 $\mathrm{m} / \mathrm{s}$ mean wind speed, $20 \%$ turbulence intensity).

\begin{tabular}{|c|c|c|}
\hline $\begin{array}{c}\text { Wind speed } \\
(\mathrm{m} / \mathrm{s})\end{array}$ & $\begin{array}{c}\partial \mathrm{T} / \partial \mathrm{p} \\
\mathrm{Nm} / \mathrm{deg} .\end{array}$ & $\begin{array}{c}\partial \mathrm{T} / \partial \mathrm{V} \\
\mathrm{Nm} /(\mathrm{m} / \mathrm{s})\end{array}$ \\
\hline 11 & -2400 & 14600 \\
\hline 13 & -8500 & 16200 \\
\hline 15 & -12400 & 19200 \\
\hline 17 & -16800 & 21600 \\
\hline 19 & -18500 & 23800 \\
\hline 21 & -22500 & 25200 \\
\hline 23 & -24200 & 25800 \\
\hline 25 & -26400 & 27200 \\
\hline
\end{tabular}

Table 1 Aerodynamic coefficients for medium-scale wind turbine 